\title{
Connecting the First Galaxies with Ultrafaint Dwarfs in the Local Group: Chemical Signatures of Population III Stars
}

\author{
Myoungwon Jeon ${ }^{1,2}$, Gurtina Besla ${ }^{1}$, and Volker Bromm ${ }^{3}$ \\ ${ }^{1}$ Department of Astronomy, University of Arizona, 933 North Cherry Avenue, Tucson, AZ 85721, USA; myjeon@email.arizona.edu \\ ${ }^{2}$ Center for Global Converging Humanities, Kyung Hee University, Korea \\ ${ }^{3}$ Department of Astronomy, University of Texas, TX 78712, USA \\ Received 2017 February 13; revised 2017 August 30; accepted 2017 September 11; published 2017 October 17
}

\begin{abstract}
We investigate the star formation history (SFH) and chemical evolution of isolated analogs of Local Group (LG) ultrafaint dwarf galaxies (UFDs; stellar mass range of $10^{2} M_{\odot}<M_{*}<10^{5} M_{\odot}$ ) and gas-rich, low-mass dwarfs (Leo $\mathrm{P}$ analogs; stellar mass range of $10^{5} M_{\odot}<M_{*}<10^{6} M_{\odot}$ ). We perform a suite of cosmological hydrodynamic zoom-in simulations to follow their evolution from the era of the first generation of stars down to $z=0$. We confirm that reionization, combined with supernova (SN) feedback, is primarily responsible for the truncated star formation in UFDs. Specifically, halos with a virial mass of $M_{\mathrm{vir}} \lesssim 2 \times 10^{9} M_{\odot}$ form $\gtrsim 90 \%$ of stars prior to reionization. Our work further demonstrates the importance of Population III stars, with their intrinsically high $[\mathrm{C} / \mathrm{Fe}]$ yields and the associated external metal enrichment, in producing low-metallicity stars $([\mathrm{Fe} / \mathrm{H}] \lesssim-4)$ and carbon-enhanced metal-poor (CEMP) stars. We find that UFDs are composite systems, assembled from multiple progenitor halos, some of which hosted only Population II stars formed in environments externally enriched by $\mathrm{SNe}$ in neighboring halos, naturally producing extremely low metallicity Population II stars. We illustrate how the simulated chemical enrichment may be used to constrain the SFHs of true observed UFDs. We find that Leo P analogs can form in halos with $M_{\mathrm{vir}} \sim 4 \times 10^{9} M_{\odot}(z=0)$. Such systems are less affected by reionization and continue to form stars until $z=0$, causing higher-metallicity tails. Finally, we predict the existence of extremely low metallicity stars in LG UFD galaxies that preserve the pure chemical signatures of Population III nucleosynthesis.
\end{abstract}

Key words: cosmology: theory - galaxies: abundances - galaxies: dwarf - galaxies: formation - galaxies: highredshift - hydrodynamics

\section{Introduction}

One of the biggest challenges in modern cosmology is to understand the first generation of stars and galaxies that formed during the cosmic Dark Ages (see, e.g., Bromm \& Yoshida 2011, for a review). They reside in observationally unexplored territory, rendering their detection one of the main goals of the upcoming next-generation facilities, such as the James Webb Space Telescope (JWST; e.g., Gardner et al. 2006) and the 30-40 m giant ground-based telescopes. The lowestmass $\left(10^{2} M_{\odot} \lesssim M_{*} \lesssim 10^{5} M_{\odot}, z=0\right)$ and most metal-poor $([\mathrm{Fe} / \mathrm{H}] \lesssim-2)$ dwarfs of the Local Group (LG), known as ultrafaint dwarfs (UFDs), have been proposed as possible descendants of the dwarf galaxies at high redshift and are thus the best place to look for the metal-poor stars that hold clues to the nature of the first generation of stars. Studying such systems enables an approach often termed "galactic archaeology," wherein local galaxies are used as fossil records of the early assembly history of the Milky Way (MW; e.g., Salvadori \& Ferrara 2009; Frebel et al. 2010; Boylan-Kolchin et al. 2015; Ricotti et al. 2016; Lapi et al. 2017; Weisz \& BoylanKolchin 2017). Here we illustrate how high-resolution cosmological simulations that follow structure formation in the smallest halos at high redshift can help link the first galaxies to their local descendants and identify the chemical signatures of the first generation of stars, the so-called Population III stars, in the stellar populations of present-day UFDs and low-mass dwarfs.

The dwarf galaxy satellites of the MW exhibit a great diversity in stellar age, metallicity, and sizes, indicating that they have experienced different star formation histories (SFHs). Specifically, more massive dwarfs $\left(M_{*}>10^{6} M_{\odot}, z=0\right)$ are characterized by extended SFHs and show large metallicity spreads, whereas the lower-mass UFDs appear to have experienced a truncated SFH, yielding uniformly ancient stellar populations, older than 11-12 Gyr (e.g., Bullock et al. 2000; Grebel \& Gallagher 2004; Brown et al. 2012, 2014; Weisz et al. 2014).

A plausible mechanism responsible for such early suppression of star formation in UFDs could be reionization, referring to the global phase transition from a neutral universe to an ionized one at $z=10-6$ (Planck Collaboration 2016). During this period of time, the radiation from stars heated the gas in the interstellar medium (ISM) and intergalactic medium (IGM), thus boosting the Jeans mass, i.e., the minimum mass to trigger gravitational instability, which in turn made it difficult for gas to collapse into halos (see, e.g., Tolstoy et al. 2009, for a review; see also Gnedin \& Kravtsov 2006; Bovill \& Ricotti 2009, 2011; Brown et al. 2012).

A new puzzle has recently been presented in the discovery of low-mass, gas-rich dwarfs in the outskirts of the MW (e.g., McQuinn et al. 2015). Dwarfs like Leo P, Leo T, Leo A, and DDO 210 have stellar masses less than $10^{6} M_{\odot}(z=0)$ but are able to retain substantial gas reservoirs $\left(M_{\mathrm{HI}} \sim 10^{5-7} M_{\odot}\right.$, $z=0$ ). Here we explore for the first time the connection between these gas-rich, low-mass dwarfs and present-day UFDs using cosmological zoom-in simulations that follow their evolution from the first generation of stars to $z=0$.

Significant theoretical progress has been made toward understanding the formation of low-mass dwarf galaxies 
(e.g., Salvadori \& Ferrara 2009; Sawala et al. 2010; Munshi et al. 2013; Simpson et al. 2013; Governato et al. 2015; Oñorbe et al. 2015; Wheeler et al. 2015; Fitts et al. 2016). However, the emphasis of these existing studies has been to understand the SFHs of dwarfs and their structural properties at $z=0$. In particular, work by Wheeler et al. (2015) and Simpson et al. (2013) used cosmological zoom-in simulations of the formation of low-mass galaxies $\left(M_{*} \lesssim 10^{6} M_{\odot}\right.$ at $\left.z=0\right)$ at high redshift to demonstrate that a combination of stellar feedback and reionization can result in the suppression of star formation in low-mass halos $\left(M_{*} \lesssim 10^{6} M_{\odot}\right.$ at $\left.z=0\right)$. However, these works only followed the chemical evolution of total metallicity, rather than individual metal species, and none tracked the metal enrichment history from Population III stars to low redshift. Furthermore, existing work concerning the chemical abundances is typically considered in an idealized setting (e.g., Revaz et al. 2009; Corlies et al. 2013; Webster et al. 2014; Bland-Hawthorn et al. 2015; Webster et al. 2015; Revaz et al. 2016). As such, the connection of the stellar populations of LG UFDs and low-mass dwarfs to those of the first generation of stars remains uncertain. We seek to address this major gap in knowledge by self-consistently following the metal enrichment and diffusion from supernovae $(\mathrm{SNe})$ from the first generation of stars in these low-mass galaxies, accounting for both reionization and their subsequent hierarchical evolution until the present day.

Ricotti \& Gnedin (2005) classify dwarf galaxies based on their SFHs into three categories: galaxies in which the bulk of stars were formed prior to reionization are classified as "true fossils," whereas "polluted fossils" refer to galaxies that have continuously formed stars beyond reionization, and "survivors" mean galaxies that formed the majority of their stars after reionization. By following the evolution of the first galaxies until the present, we will be able to establish the expected degree of "pollution" in UFDs and furthermore quantify the significance of late-time accretion events to their present-day chemical properties. True fossils, in particular, could offer more stringent insight on the nature of the first generation of stars, preserving their chemical signatures since these galaxies are not likely to undergo significant star formation after reionization.

The SN feedback from Population III stars drove the gas out of minihalos, thus polluting the surrounding IGM with the metals (e.g., Kitayama \& Yoshida 2005; Ritter et al. 2012; Jeon et al. 2014). The second generation, called Population II stars, were born out of the gas thus shaped by Population III SNe, and as a result, they might preserve the chemical signatures of the Population III stars. Unlike Population III stars, the lower mass of Population II stars can allow them to survive until today. Therefore, investigating the chemical abundance patterns of Population II stars may inform us of the star formation at early times in UFDs and low-mass dwarfs. In particular, the abundances of alpha elements compared to iron can tell us about the timescale over which stars have formed and about the corresponding star formation efficiency.

To date, a complete theoretical understanding of chemical abundances in UFD stellar populations, in a full cosmological context, and considering the detailed enrichment from early Population III and Population II stars, is still lacking. This study is the first to self-consistently follow these processes from high redshift to the present day, allowing us to constrain the expected signatures of Population III stars in the stellar populations of current UFDs and low-mass dwarfs.
Here we present the results of a suite of cosmological, highresolution zoom-in simulations that trace the chemical and mass evolution of low-mass dwarf galaxies (UFD mass analogs) from high redshifts down to $z=0$. In this work, we have two main goals: (1) to investigate the role of reionization and SN feedback in the SFHs of UFD and low-mass, gas-rich dwarf analogs, and (2) to compare the resulting stellar abundance patterns with observational data from present-day UFDs, highlighting signatures of chemical enrichment from Population III stars. We will discuss the structural properties of the simulated dwarfs (e.g., velocity dispersions and sizes) in an upcoming paper.

The most important difference of this work from previous studies is the ab initio inclusion of early star formation and enrichment. In addition, for the first time, we derive detailed chemical abundance patterns for individual stars from realistic cosmological simulations, to be compared with stellar archaeological data. Our hydrodynamical simulations are nicely complemented by the recent dark-matter-only (DM-only) investigation of Griffen et al. (2016). Both treatments employ cosmological initial conditions, with the latter focusing on representing the realistic large-scale density field of the LG environment, while our work includes the detailed physics of the baryonic component. L. Corlies et al. (2017 private communication) consider the SFHs and metallicity distribution functions (MDFs) of a range of halos within a fully simulated cosmological box as opposed to zooming in on specific halos. However, the computationally intensive feedback methods implemented restrict the simulation to a small volume and can only be run to $z=7$.

The outline of the paper is as follows. Our numerical methodology is described in Section 2, and the simulation results are presented in Section 3. We discuss the limitations of this work in Section 4. Finally, our main findings are summarized in Section 5. For consistency, all distances are expressed in physical (proper) units unless noted otherwise.

\section{Numerical Methodology}

In this section, we present numerical methods we adopt for the simulations. At first, we explain initial conditions, followed by the cosmological and hydrodynamical parameters in Section 2.1. The implemented chemistry, cooling, and UV background are presented in Section 2.2. In Section 2.3, we describe the star formation recipe for Population III and Population II stars. The accompanied chemical feedback of $\mathrm{SNe}$ from both populations is discussed in Section 2.4. Finally, we show how we include thermal feedback from $\mathrm{SNe}$ in Section 2.5. Note that possible caveats and limitations of the methods described here are pointed out in Section 4.

\subsection{Simulation Setup}

We have performed a suite of hydrodynamic zoom-in simulations using a modified version of the $N$-body/TreePM smoothed particle hydrodynamics (SPH) code GADGET (Springel et al. 2001; Springel 2005). As cosmological parameters, we adopt a matter density of $\Omega_{\mathrm{m}}=$ $1-\Omega_{\Lambda}=0.265$, a baryon density of $\Omega_{\mathrm{b}}=0.0448$, a present-day Hubble expansion rate of $H_{0}=71 \mathrm{~km} \mathrm{~s}^{-1} \mathrm{Mpc}^{-1}$, a spectral index $n_{\mathrm{s}}=0.963$, and a normalization $\sigma_{8}=0.801$ (Komatsu et al. 2011), consistent with the most recent constraints from the Planck satellite (Planck Collaboration 2016). 

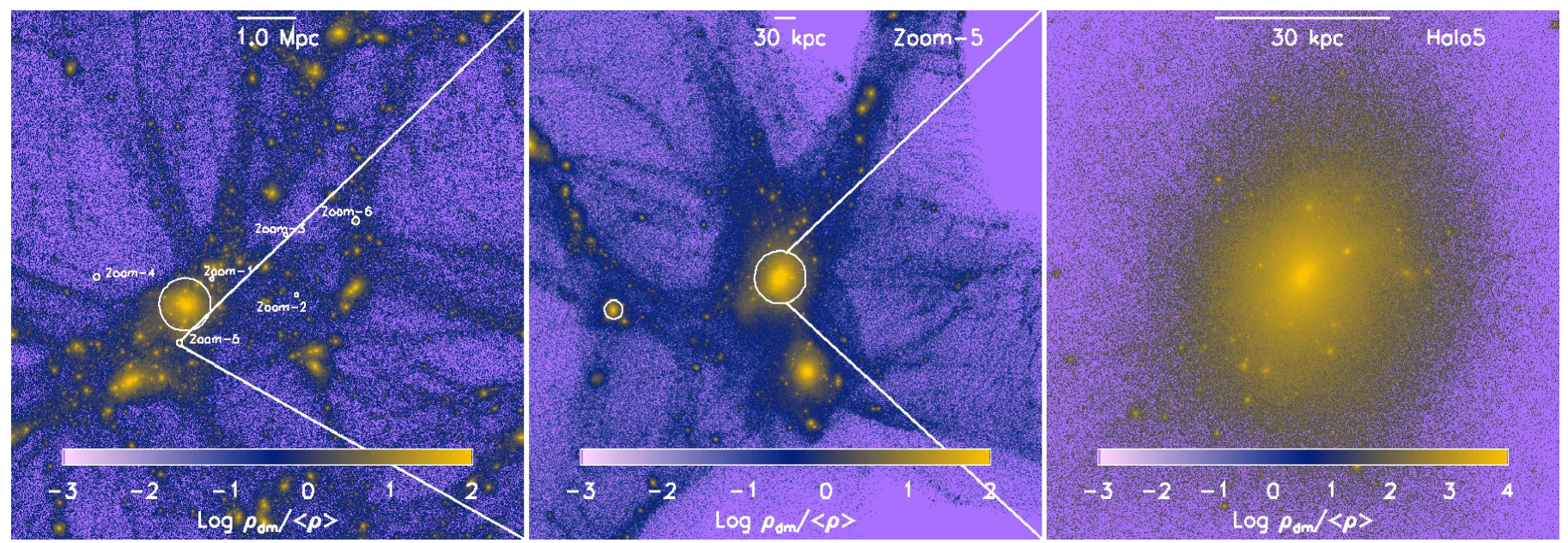

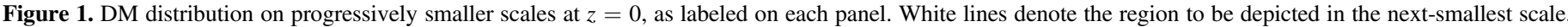

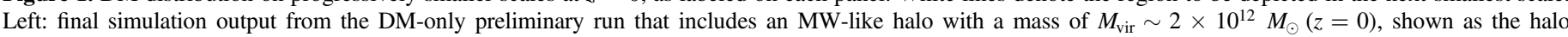

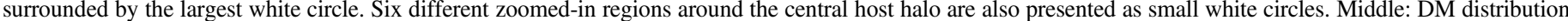

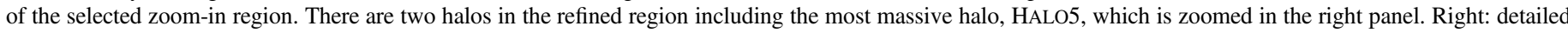
look of HALO5. The virial radius of the halo is $\sim 30 \mathrm{kpc}$ at $z=0$.

Table 1

Characteristics of the Simulated UFD and Gas-rich Dwarf Analogs at $z=0$

\begin{tabular}{|c|c|c|c|c|c|c|c|c|c|c|c|c|}
\hline $\begin{array}{l}\text { Halo } \\
\text { Unit }\end{array}$ & $\begin{array}{c}M_{\mathrm{vir}} \\
\left(10^{9} M_{\odot}\right)\end{array}$ & $\begin{array}{c}r_{\mathrm{v}} \\
(\mathrm{kpc})\end{array}$ & $\begin{array}{c}M_{*} \\
\left(10^{4} M_{\odot}\right)\end{array}$ & $\begin{array}{c}D_{\mathrm{h}} \\
(\mathrm{Mpc})\end{array}$ & $\begin{array}{c}f_{\mathrm{b}} \\
(\%)\end{array}$ & $\begin{array}{l}r_{1 / 2}^{*} \\
(\mathrm{pc})\end{array}$ & $\begin{array}{c}{[\mathrm{Fe} / \mathrm{H}]} \\
\ldots\end{array}$ & $\begin{array}{c}{[\alpha \overline{/ F e}]} \\
\ldots\end{array}$ & $\begin{array}{c}\sigma_{*} \\
\left(\mathrm{kms}^{-1}\right)\end{array}$ & $\begin{array}{c}M_{\text {gas }} \\
\left(10^{6} M_{\odot}\right)\end{array}$ & $\begin{array}{c}M_{\mathrm{H} \mathrm{I}, \mathrm{w}} / M_{\mathrm{H} \mathrm{I}, \mathrm{c}} \\
\left(10^{5} M_{\odot}\right)\end{array}$ & $\begin{array}{c}\mathrm{SF}_{\text {trun }} \\
\cdots\end{array}$ \\
\hline Halo1 & 1.53 & 23.7 & 4.3 & 0.6 & 0.08 & 345 & -2.63 & 0.52 & 6.4 & 1.30 & $\ldots$ & Yes \\
\hline Halo2 & 1.53 & 23.5 & 3.8 & 2.0 & 0.07 & 320 & -2.25 & 0.44 & 6.0 & 1.15 & $\ldots$ & Yes \\
\hline Halo3 & 1.60 & 23.9 & 8.2 & 2.1 & 0.1 & 296 & -2.28 & 0.52 & 6.7 & 1.67 & $\ldots$ & Yes \\
\hline Halo4 & 2.21 & 26.6 & 13.0 & 1.9 & 0.96 & 513 & -2.45 & 0.54 & 11.2 & 1.13 & $\ldots$ & No \\
\hline Halo5 & 3.15 & 29.9 & 20.0 & 0.9 & 0.05 & 479 & -2.27 & 0.53 & 9.9 & 1.58 & $\ldots$ & No \\
\hline Halo6 & 3.95 & 32.1 & 88.6 & 3.7 & 0.9 & 438 & -1.23 & 0.47 & 11.6 & 26 & $44 / 0.59$ & No \\
\hline
\end{tabular}

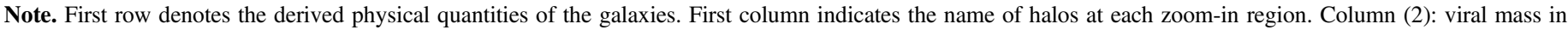

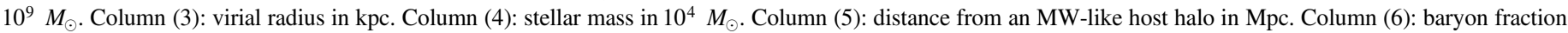

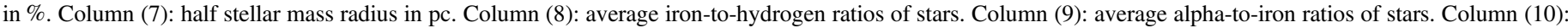

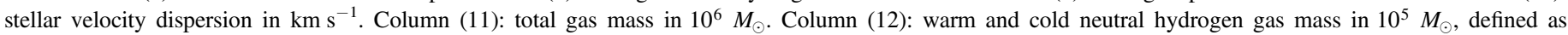

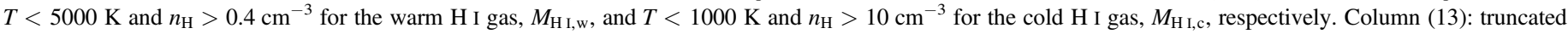
star formation after reionization.

The initial conditions are generated using the cosmological initial conditions code MUSIC (Hahn \& Abel 2011). As a preliminary run, we perform a DM-only simulation using $128^{3}$ particles in an $L=6.25 h^{-1}$ comoving Mpc box. Then, we identify six galaxies with a mass of $M_{\mathrm{vir}} \sim 10^{9} M_{\odot}$ at $z=0$ around an MW-size halo $\left(M_{\mathrm{vir}} \sim 2 \times 10^{12} M_{\odot}\right.$; see Figure 1). We confirm that the selected dwarfs have maintained an isolated position, outside of the virial radius of the MW-size host halo throughout their evolution. We conduct four consecutive refinements for all particles within $\sim 2 R_{\text {vir }}$ of the selected lowmass halos at $z=0$, where $R_{\mathrm{vir}}$ is a virial radius, as marked in Figure 1, defined as the radius at which the mass density is 200 times higher than the average density of the universe. The resultant effective resolution is $2048^{3}$, giving rise to DM and gas masses in the most refined region of $m_{\mathrm{DM}} \approx 2000 M_{\odot}$ and $m_{\mathrm{SPH}} \approx 495 M_{\odot}$, respectively.

The properties of the selected halos (UFD analogs) at $z=0$ are listed in Table 1 and discussed in detail in Section 3. The most massive halo, HALO6, is our prime candidate analog of gas-rich, low-mass dwarfs like Leo P, Leo A, Leo T, and DDO 210. This will be discussed in more detail in Section 3.2.
We find that in order to minimize computational cost, we preferentially choose halos in relatively isolated regions, distributed from 0.6 to $2.1 \mathrm{Mpc}$ from the center of the MWsize halo, which is illustrated as the largest white circle in the left panel of Figure 1. This choice naturally allows us to exclude other possible processes that might affect the SFHs and chemical properties of the dwarfs, such as tidal interactions and ram pressure stripping. We fix the softening lengths for DM and star particles as $\epsilon_{\mathrm{DM}}=40 \mathrm{pc}$ at all redshifts. We use adaptive softening length for the gas particles, where the softening length is proportional to the SPH kernel length with a minimum value of $\epsilon_{\mathrm{gas}}$ min $=2.8 \mathrm{pc}$.

\subsection{Chemistry, Cooling, and UV Background}

We solve the coupled, nonequilibrium rate equations every time step for the primordial chemistry from nine atomic and molecular species $\left(\mathrm{H}, \mathrm{H}^{+}, \mathrm{H}^{-}, \mathrm{H}_{2}, \mathrm{H}_{2}^{+}, \mathrm{He}, \mathrm{He}^{+}, \mathrm{He}^{++}\right.$, and $e^{-}$), as well as from the three deuterium species $\mathrm{D}, \mathrm{D}^{+}$, and $\mathrm{HD}$ (Glover \& Jappsen 2007) in the presence of the cosmic UV/ $\mathrm{X}$-ray background provided by Haardt \& Madau (2012). The UV background is implemented in terms of ionization and heating rates of $\mathrm{HI}$, He I, and $\mathrm{He}$ II as a function of redshift. 
Such background is an important factor in influencing the SFHs, in that the early presence of UV radiation is likely to suppress star formation early on. Here we begin to include a UV background at $z=7$ and linearly increase its strength until $z=6$. At lower redshifts, we incorporate the background at full amplitude, assuming that reionization is complete at $z=6$ (e.g., Gunn \& Peterson 1965; Fan et al. 2006).

Self-shielding of the dense gas is included such that the UV background is attenuated as a function of $\exp \left(-N_{\mathrm{HI}} \bar{\sigma}_{\text {ion }}\right)$, where $N_{\mathrm{H} \mathrm{I}}=x n_{\mathrm{H} \mathrm{I}}$. Here $x=h$ is the SPH kernel size, $n_{\mathrm{H} \mathrm{I}}$ the neutral hydrogen number density, and $\bar{\sigma}_{\text {ion }}$ the frequencyaveraged photoionization cross section for $\mathrm{H}$ I. In addition, the photodissociation of molecular hydrogen, $\mathrm{H}_{2}$, by the UV radiation in the Lyman-Werner $(\mathrm{LW})$ band $(11.2-13.6 \mathrm{eV})$ is considered (e.g., Abel et al. 1997), with a rate $k_{\mathrm{LW}}=$ $1.38 \times 10^{-12} \mathrm{~s}^{-1} J_{21}$. Here the normalized LW mean intensity is $J_{21}=\bar{J}_{\nu} /\left(10^{-21} \mathrm{erg} \mathrm{Hz}^{-1} \mathrm{~s}^{-1} \mathrm{~cm}^{-2} \mathrm{sr}^{-1}\right)$, with $\bar{J}_{\nu}(z)$ being the average mean intensity in the LW band, calculated from the spectra in Haardt \& Madau (2011). The treatment for photodissociation of deuterated hydrogen HD is identical to that of $\mathrm{H}_{2}$.

We consider all relevant primordial cooling processes such as $\mathrm{H}$ and $\mathrm{He}$ collisional ionization, excitation and recombination cooling, bremsstrahlung, inverse Compton cooling, and collisional excitation cooling of $\mathrm{H}_{2}$ and HD. Additionally, gas cooling by metal species such as carbon, oxygen, silicon, magnesium, neon, nitrogen, and iron is taken into account under equilibrium conditions. Specifically, we employ the method implemented by Wiersma et al. (2009a), where the cooling rates are computed element by element from precomputed tables based on the photoionization code CLOUDY (Ferland et al. 1998), by interpolating the value as a function of density, temperature, and metallicity. Note that we ignore $\mathrm{H}_{2}$ formation via dust and also dust cooling for the following reasons: (1) at high redshifts, $z>7$, the amount of dust is not sufficient to be a dominant source of $\mathrm{H}_{2}$ formation, particularly in small systems $\left(M_{*} \lesssim 10^{6} M_{\odot}\right)$; and (2) at low redshifts, metal cooling dominates over $\mathrm{H}_{2}$ cooling.

\subsection{Star Formation Physics}

The first stars are predicted to form out of primordial gas inside minihalos $\left(M_{\mathrm{vir}} \sim 10^{5}-10^{6} M_{\odot}\right)$ at $z \gtrsim 15$ (see, e.g., Bromm 2013, for a review; see also Haiman et al. 1996; Tegmark et al. 1997). The Population III initial mass function (IMF) is still a subject of debate (e.g., Bromm 2013). The final mass of Population III stars is determined by the complex interplay between protostellar UV feedback, gas accretion through a self-gravitating circumstellar disk, and its fragmentation via gravitational instability (e.g., McKee \& Tan 2008; Hosokawa et al. 2011; Stacy et al. 2012). Recent 2D and 3D radiation hydrodynamic simulations, including stellar feedback from a protostar, predict that the mass of Population III stars is broadly distributed from $m_{*} \lesssim 1 M_{\odot}$ to $m_{*} \sim 1000 M_{\odot}$ (e.g., Hirano et al. 2014; Susa et al. 2014; Hosokawa et al. 2016; Stacy et al. 2016). The statistical study by Hirano et al. (2015) suggests that the characteristic mass of Population III stars also sensitively depends on the photodissociation from external far-UV (FUV) radiation, emitted by neighboring Population III stars.

Due to the limited numerical resolution, individual stars cannot be resolved, and we instead allow Population III stars to form as a single star cluster. We assume a top-heavy initial mass function (IMF) with a functional form of $\phi_{\text {Pop III }}(m)=$ $d N / d \log m=$ const. and a mass range of $\left[m_{0}, m_{1}\right]=$ $[10,150] M_{\odot}$. Once a gas particle exceeds a threshold density of $n_{\mathrm{H}, \text { th }}=100 \mathrm{~cm}^{-3}$, the highest-density SPH particle is converted into a collisionless star particle with a $100 \%$ conversion efficiency, forming $M_{*} \approx 500 M_{\odot}$ in Population III stars at once. We note that the adapted threshold density is somewhat lower than the characteristic value, $n_{\mathrm{H} \text {, th }}=$ $10^{4} \mathrm{~cm}^{-3}$, where gravitational instability is triggered in primordial star formation (e.g., Abel et al. 2002; Bromm et al. 2002). In an effort to reduce computational costs, we avoid implementing such a high threshold density. However, our choice of $n_{\mathrm{H}, \mathrm{th}}=100 \mathrm{~cm}^{-3}$ is reasonable given that, above $n_{\mathrm{H}}=$ $10^{3} \mathrm{~cm}^{-3}$, the Population III star formation time is insensitive to any further increase in threshold density (Muratov et al. 2013).

Stars are formed from gas clouds at a rate $\dot{\rho}_{*}=\rho_{\text {th }} / \tau_{*}$, where $\tau_{*}=\tau_{\mathrm{ff}} / \epsilon_{\mathrm{ff}}$ is the star formation timescale, $\tau_{\mathrm{ff}}=\left[3 \pi /\left(32 G \rho_{\mathrm{th}}\right)\right]^{1 / 2}$ is the freefall time at the threshold density $\rho_{\mathrm{th}}$, and $\epsilon_{\mathrm{ff}}$ is the star formation efficiency per freefall time (Schmidt 1959). The star formation efficiency for Population III stars has yet to be pinned down precisely (e.g., Hirano et al. 2015; Stacy et al. 2016). Using a global baryon fraction of $f_{\mathrm{b}}=0.168$, we set $\epsilon_{\mathrm{ff} \text {, Pop III }}=$ $M_{\text {char,Pop III }} /\left(M_{\text {vir }} \times f_{\mathrm{b}}\right)$, where $M_{\text {char,Pop III }}$ is the characteristic mass of Population III stars, leading to $\epsilon_{\mathrm{ff}, \text { Pop III }} \sim 0.01$. We note that this value is similar to a typical efficiency in local star formation. Then, an SPH particle is stochastically converted into a collisionless star particle in a time interval $\Delta t$, if a random number is smaller than $\min \left(\Delta t / \tau_{*}, 1\right)$, to follow a given distribution $\dot{\rho}_{*}=\rho_{\text {th }} / \tau_{*}$. The star formation timescale is then given by

$$
\tau_{*}=\frac{\tau_{\mathrm{ff}}\left(n_{\mathrm{H}, \mathrm{th}}\right)}{\epsilon_{\mathrm{ff}}} \sim 400 \operatorname{Myr}\left(\frac{n_{\mathrm{H}, \mathrm{th}}}{100 \mathrm{~cm}^{-3}}\right)^{-1 / 2} .
$$

Once pristine gas is enriched with metals dispersed by SN explosions of the first generation of stars, low-mass, long-lived, second-generation Population II stars are formed out of the metal-polluted gas clouds. For Population II star formation, we employ the same star formation recipe as for Population III, with an identical star formation efficiency, $\epsilon_{\mathrm{ff} \text {, Pop II }}=0.01$, but adding an additional metallicity criterion. If the metallicity of a gas particle, eligible for star formation, exceeds the critical metallicity, $Z_{\text {crit }}=10^{-5.5} Z_{\odot}$, for the transition from Population III to Population II, we form a Population II star cluster. The choice of $Z_{\text {crit }}=10^{-5.5} Z_{\odot}$ is motivated by dust continuum cooling (e.g., Omukai 2000; Bromm et al. 2001; Schneider \& Omukai 2010; Safranek-Shrader et al. 2016), where dust cooling is responsible for further gas fragmentation at high densities, $n_{\mathrm{H}} \gtrsim 10^{16} \mathrm{~cm}^{-3}$, enabling the formation of low-mass stars. For the Population II IMF, we use a Chabrier IMF over the mass range of $[0.1-100] M_{\odot}$.

\subsection{Chemical Feedback}

In this section, we describe how we implement stellar yields from SN explosions and metal diffusion into the ISM and IGM. We use an implementation of chemodynamics where enrichment by winds from asymptotic giant branch (AGB) stars and by Type II and Type Ia SNe is incorporated (Wiersma et al. 2009b). At every time step, the mass of 11 individual elements from dying stars is computed and released into the neighboring medium, followed by subsequent diffusive mixing in the ISM and IGM. 
We have, for the first time, applied the metal diffusion scheme to understand the evolution of chemical abundances of dwarf galaxies in the full cosmological context. We briefly outline the procedure here.

\subsubsection{Stellar Yields: Population III Stars}

The low atmospheric opacities due to the absence of metals renders mass loss by stellar winds from primordial stars almost negligible (e.g., Kudritzki \& Puls 2000). Therefore, the final fate of Population III stars is determined by their initial mass. For example, massive stars in the mass range between 10 and $40 M_{\odot}$ end their lives as conventional CCSNe, some of which are hypernovae in the range of $25 M_{\odot} \lesssim m_{*} \lesssim 40 M_{\odot}$. Very massive stars within the particular mass range of $140 M_{\odot} \lesssim m_{*} \lesssim 260 M_{\odot}$ are expected to experience PISNe, powered by electron-positron pair creation. In the latter case, after central helium burning, the core temperature becomes sufficiently high to abundantly produce electron-positron pairs, such that the star begins to collapse owing to the sudden drop in radiation pressure support. The rapid contraction, in turn, triggers implosive oxygen and silicon burning, which produces enough energy to completely disrupt the star, leaving no remnant behind. If Population III stars are more massive than $m_{*}>260 M_{\odot}$, they are expected to undergo direct collapse into a black hole without any explosion since the thermonuclear energy release is not sufficient to reverse the implosion.

Nucleosynthetic yields of CCSNe from Population III progenitor stars and their remnant masses are provided by Heger \& Woosley (2010), comprising progenitor masses of $10-100 M_{\odot}$, and exploring a range of explosion energies between $0.3 \times 10^{51} \mathrm{erg}$ and $10^{52} \mathrm{erg}$. Specifically, we use yields for SN explosion energy of $1.2 \times 10^{51} \mathrm{erg}$, in the absence of rotation. For PISNe, we adapt the yields given in Heger \& Woosley (2002). It should be noted that the effects of stellar rotation and magnetic fields are not considered. For example, some recent studies suggest that under the influence of stellar rotation the lowest mass resulting in a PISN can be shifted down to $75 M_{\odot}$ (e.g., Chatzopoulos \& Wheeler 2012).

\subsubsection{Stellar Yields: Population II Stars}

Stars experience strong mass loss at the end of their lives, corresponding to the AGB or SN phase. The lifetime of a star is defined as the time when a star leaves the main sequence and enters its red giant phase. Here we employ metallicitydependent tables of stellar lifetimes, covering values from $Z=0.0004$ to $Z=1.0$ (Portinari et al. 1998). Intermediatemass stars $\left(0.8 M_{\odot} \lesssim m_{*}<8 M_{\odot}\right)$ lose up to $60 \%$ of their mass during the terminal AGB stage. The corresponding yields are taken from Marigo (2001), where stars as massive as $5 M_{\odot}$ are covered. Due to the low wind velocity compared to the velocity dispersion in the ISM, the kinetic energy input from AGB winds is neglected.

Substantial amounts of metals are ejected by Type II SNe when massive stars $\left(m_{*} \gtrsim 8 M_{\odot}\right)$ explode at the end of their life. The yields from Type II SNe are from Portinari et al. (1998), in which mass loss on the main sequence is considered. They provide a yield set that is derived in a self-consistent manner with the AGB yields, in terms of stellar lifetimes and mass range considered. Due to our short hydrodynamic time steps, $\Delta t=0.01-0.1 \mathrm{Myr}$, compared to the timescale over which Type II SNe occur, metals are released through multiple time steps after the most massive star in a stellar cluster undergoes an $\mathrm{SN}$ explosion, followed by subsequent explosions of less massive stars. The energy associated with Type II $\mathrm{SNe}$ is distributed onto neighboring particles as thermal energy, composing a total of $E_{\mathrm{SN} \text {, Pop II }}=8.6 \times 10^{51} \mathrm{erg}$. This value is obtained by integrating over a Chabrier IMF in the mass range of $[6,100] M_{\odot}$ for a Population II cluster. The SN energy is deposited at once in a single time step at the end of the most massive star's life in a Population II cluster. A detailed description is to follow in Section 2.5.

The progenitor mass of a Type Ia $\mathrm{SN}$ is thought to be between 3 and $8 M_{\odot}$. Once the mass of a white dwarf remnant exceeds the Chandrasekhar mass, by either mass transfer from a companion star or the merger of two white dwarfs, a Type Ia $\mathrm{SN}$ is triggered. Due to the large uncertainty attributed to the details of binary evolution, determining the Type Ia SN rate is highly complex, compared to the mass release by Type II SNe and AGB stars, where mass loss simply occurs at the end of the progenitor's life. We employ empirical delay time functions (e.g., Barris \& Tonry 2006; Förster et al. 2006), expressed as $e$ folding times, $\eta(t)=e^{-t / \tau_{\mathrm{Ia}}} / \tau_{\mathrm{Ia}}$, where $\tau_{\mathrm{la}}=2 \mathrm{Gyr}$ is the characteristic delay time. The SN Ia rate at a given time step $\Delta t$ is then $N_{\mathrm{SN} \mathrm{Ia}}(t ; t+\Delta t)=a \int_{t}^{t+\Delta t} f_{\mathrm{wd}}\left(t^{\prime}\right) \eta\left(t^{\prime}\right) d t^{\prime}$, where $a=0.01$ is a normalization parameter and $f_{\mathrm{wd}}$ is the number of stars that have evolved into white dwarfs per unit stellar mass (Mannucci et al. 2006). The Type Ia SN yields, based on the explosion of a Chandrasekhar-mass carbon-oxygen white dwarf, are taken from the spherically symmetric "W7" model (Thielemann et al. 2003). The corresponding SN energy is again distributed onto neighboring particles as thermal energy, but this feedback is likely to be less effective in disturbing the surrounding gas because the energy injection is distributed over billions of years.

\subsubsection{Metal Diffusion}

After metals from AGB stellar winds, from Type II and Type Ia SNe, and from PISNe are released, they should be transported into the ISM and IGM. However, owing to the lack of intrinsic mass flux between SPH particles, the implementation of metal transfer in SPH simulations is nontrivial. Commonly, a "particle metallicity" has been widely used, where metals are locked up into the initial neighboring gas particles, resulting in a very compact metal distribution around an SN explosion site. A new, improved implementation has been suggested by Wiersma et al. (2009b), where gas metallicity is computed in terms of density $Z_{\mathrm{sm}}=\rho_{\mathrm{Z}} / \rho$. This smoothed metallicity is suitable in simulating galaxy formation because gas cooling, one of the key factors in star formation, depends on gas density. Thus, using the smoothed metallicity, derived with the SPH kernel formalism, allows one to compute metallicity-dependent gas cooling in a more consistent way. Also, the smoothed metallicity partially accounts for the spreading of metals. The method we adopt here, on the other hand, is a diffusion-based metallicity implemented by Greif et al. (2009), where the mixing efficiency on unresolved scales is determined by the physical properties on the scale of the SPH smoothing kernel (Klessen \& Lin 2003).

Initially, the ejected metals are distributed among the neighboring gas particles, $N_{\text {ngb }}=48$, giving rise to initial particle metallicities,

$$
Z_{i}=\frac{m_{\text {metal,i }}}{m_{\mathrm{SPH}}+m_{\text {metal,i }}} .
$$


Then, the metals are transported by solving the diffusion equation, written in the form

$$
\frac{d c}{d t}=\frac{1}{\rho} \nabla \cdot(D \nabla c)
$$

where $c$ is the concentration of a fluid per unit mass, corresponding to the total gas metallicity in this work. We also track individual metal species element by element such as $\mathrm{C}, \mathrm{O}, \mathrm{Si}, \mathrm{Mg}, \mathrm{Ne}, \mathrm{Ni}$, and $\mathrm{Fe} . \mathrm{D}$ is the diffusion coefficient defined as $D=2 \rho \tilde{v} \tilde{l}$, where the length scale, $\tilde{l}$, is comparable to the smoothing length of the SPH kernel, $\tilde{l}=h$, and $\rho$ is the gas density. The velocity dispersion within the kernel, $\tilde{v}$, is given by

$$
\tilde{v}_{i}^{2}=\frac{1}{N_{\text {ngb }}} \sum_{j}\left|v_{i}-v_{j}\right|^{2} .
$$

Here $v_{i}$ and $v_{j}$ are the velocities of particles $i$ and $j$ within the kernel. Efficient metal mixing is achieved owing to the increased velocity dispersion of the gas as the forward shock from an SN explosion passes by. It should be mentioned that this method assumes that motions in the resolved scales cascade down to unresolved scales within which the gas is homogeneously mixed. The resulting velocity field is driven by a homogeneously and isotropically turbulent medium, meaning that three-dimensional structures within the medium are not considered.

\subsection{Thermal Feedback}

During the main-sequence stage, Population III and Population II stars emit photons that ionize and heat the surrounding medium, but in this work we do not include photoheating and radiation pressure from stars for the sake of feasibility. It would be computationally prohibitive to follow galaxy evolution over time down to $z=0$ while self-consistently solving the radiative transfer equation for our highly resolved star-forming regions. Once a massive Population III or Population II star dies, exploding as a Type II SN, the explosion energy is injected into the surrounding medium, destroying dense gas and thus suppressing subsequent star formation. Here we insert SN energy as thermal energy, distributed onto neighboring gas particles. The common problem regarding the thermal energy method is that gas particles heated by an $\mathrm{SN}$ explosion radiate their energy away too quickly, before making SN feedback effective. This is in part due to the unphysical absence of photoheating in our current simulations, which would otherwise act to photoevacuate gas prior to the SN explosion, rendering the surrounding medium too dense.

Another problem is that $\mathrm{SN}$ energy is deposited onto too much gas mass, owing to the limited resolution. In reality, one CCSN is triggered out of every $\sim 100 M_{\odot}$ in stars, and the SN energy is carried by $<<100 M_{\odot}$ of ejecta, while in cosmological simulations the mass of neighboring gas particles that receive the SN energy is much larger than the physical ejecta mass. Consequently, temperatures in the heated gas remain below $T \sim 10^{6} \mathrm{~K}$, where the cooling time is too short. Therefore, one widely used way to circumvent the overcooling problem is disabling gas cooling for a few tens of Myr. In our simulations, on the other hand, we follow the strategy proposed by Dalla Vecchia \& Schaye (2012), which assigns temperatures above $10^{7.5} \mathrm{~K}$ to the heated particles, thus preventing the gas from radiatively losing its energy too quickly. We briefly summarize this approach here.

The temperature jump of gas particles that receive SN energy is given by

$$
\begin{aligned}
\Delta T= & (\gamma-1) \frac{\mu m_{\mathrm{H}}}{k_{\mathrm{B}}} \epsilon_{\mathrm{SN}} \frac{M_{*}}{m_{\mathrm{g}, \text { heat }}} \\
= & 4.34 \times 10^{7} \mathrm{~K}\left(\frac{n_{\mathrm{SN}}}{1.736 \times 10^{-2} M_{\odot}^{-1}}\right)\left(\frac{\mu}{0.6}\right) \\
& \times E_{51} \frac{M_{*}}{m_{\mathrm{g}, \text { heat }}},
\end{aligned}
$$

where $\epsilon_{\mathrm{SN}}=n_{\mathrm{SN}} E_{51} \times 10^{51} \mathrm{erg}$ is the total available $\mathrm{SN}$ energy per unit stellar mass and $E_{51} \times 10^{51} \mathrm{erg}\left(E_{51}=1\right)$ is the available energy from a single CCSN event. The number of stars per unit stellar mass ending their lives as Type II SNe is defined as $n_{\mathrm{SN}}=\int_{m_{0}}^{m_{1}} \phi(m) d m$, where $m_{0}$ and $m_{1}$ are the minimum and maximum initial mass of stars eligible for SN explosion, respectively, and $\phi(m)$ is a given IMF. For Population III clusters, the number densities of CCSNe and PISNe per stellar mass are $n_{\mathrm{CCSN}, \text { Pop III }}=1.2 \times 10^{-2} M_{\odot}^{-1}$ $\left(\left[m_{0}, m_{1}\right]=[11,40] M_{\odot}\right)$ and $n_{\mathrm{PISN}, \text { Pop III }}=4.9 \times 10^{-4} M_{\odot}^{-1}$ $\left(\left[m_{0}, m_{1}\right]=[140,150] M_{\odot}\right)$, respectively. The latter means that typically two PISN events happen in the $500 M_{\odot}$ Population III cluster, $N_{\text {PISN }}=n_{\text {PISN,Pop III }} M_{* \text {,Pop III }} \sim 0.24$. For Population II clusters with the assumed Chabrier IMF, the number density of $\mathrm{CCSNe}$ is $n_{\mathrm{CCSN}, \text { Pop II }}=1.73 \times 10^{-2} M_{\odot}^{-1} \quad\left(\left[m_{0}, m_{1}\right]=\right.$ $\left.[8,100] M_{\odot}\right)$. The total available SN energy from a single Population III and Population II cluster is $E_{\mathrm{SN}}=\left(\epsilon_{\mathrm{CCSN}, \text { Pop III }}+\right.$ $\left.\epsilon_{\text {PISN, Pop III }}\right) \times M_{* \text {,Pop III }}=2.75 \times 10^{52} \mathrm{erg}$, where $\epsilon_{\text {PISN, Pop III }}=$ $n_{\mathrm{PISN} \text {,Pop III }} \times\left(7 \times 10^{52}\right)$ erg and $\epsilon_{\mathrm{CCSN} \text {, Pop II }} \times M_{* \text {,Pop II }}=$ $8.5 \times 10^{51} \mathrm{erg}$, respectively. In order to assure that the heated gas reaches above $10^{7} \mathrm{~K}$, making the thermal $\mathrm{SN}$ feedback effective in the surrounding medium, we release the SN energy at once when the most massive star in a cluster undergoes an SN explosion.

In the standard SPH thermal feedback implementation, SN energy is normally distributed onto neighboring SPH particles, where $N_{\text {neigh }}=48$. If we heat all the neighboring particles, then the total heated gas mass is $m_{\mathrm{g}, \text { heat }}=m_{\mathrm{SPH}} N_{\text {neigh }}=2.3 \times$ $10^{4} M_{\odot}$ and it renders the ratio $M_{*} / m_{\mathrm{g} \text {,heat }}=0.02$ for the Population III and Population II clusters. Consequently, the neighboring gas particles would achieve a temperature jump lower than $\Delta T \sim 10^{7.5} \mathrm{~K}$ by an order of magnitude, making SN feedback incapable of impacting the gas. In order to increase the temperature jump, we decrease the ratio $M_{*} / m_{\mathrm{g} \text {,heat }}$ by reducing the number of neighboring heated particles to a single particle, the closest one around a stellar cluster. Heating a single gas particle ensures that the ratio $M_{*} / m_{\mathrm{g} \text {,heat }}$ is of order unity.

Additionally, we use a time step limiter such that the ratio of time steps of neighboring SPH particles cannot be larger than a given factor, here adopting a value of 4 (Saitoh \& Makino 2009; Durier \& Dalla Vecchia 2012). The implementation is essential for the correct treatment of SN explosions, especially for highresolution multiphase simulations with individual time steps, where the hot gas $\left(T>10^{7.5} \mathrm{~K}\right)$ is located near cold, dense gas $\left(T<10^{4} \mathrm{~K}\right)$, resulting in a large difference in their time steps 
that eventually could lead to a large integration error. Also, we consider a time step update where all neighboring particles around an SN explosion site become active particles at the time of energy injection (Dalla Vecchia \& Schaye 2012), allowing them to immediately react to a sudden SN event.

\section{Simulation Results}

In the following, we present our simulation results concerning the chemical properties of low-mass reionization relics, in comparison to the observed properties of the lowest-mass galaxies about the MW. In Section 3.1, we consider the SFH of the simulated galaxies over cosmic time down to $z=0$. In Section 3.2, we focus on HALO6, the most massive simulated halo, in order to discuss residual gas at $z=0$ and analogs of low-mass, gas-rich dwarfs, such as Leo P, Leo A, Leo T, and DDO 210. Next, we explain the general trend of metal yields from Population III and Population II stars in Section 3.3 and the resulting chemical abundances of the UFDs in Section 3.4. We further discuss how to provide insight into the SFHs and accretion timescales of the observed LG UFDs by comparing with our simulated UFD analogs. Finally, in Section 3.6, we present the global galaxy properties at $z=0$ and compare our results with other theoretical studies and observations. All the simulated dwarfs reside at distances from 0.6 to $3.7 \mathrm{Mpc}$ from the central galaxy at $z=0$ (see Figure 1). As such, environmental factors (tides, ram pressure stripping, UV from central galaxy) are not relevant to the evolution of these galaxies.

\subsection{Star Formation History}

\subsubsection{SFH of the Primary Dwarf Halo (i.e., the Most Massive Halo in Its Merger History)}

Figure 2 shows the time evolution of the zoomed-in lowmass halos in each refined region (clockwise from upper left): the total and gas mass, the maximum hydrogen number density, cumulative fractional SFH, and star formation rate (SFR). These quantities are computed based on the properties of particles within the virial volume of the halos. Most of the halos begin to grow from minihalos with a mass of $M_{\text {vir }}=10^{5}-10^{6} M_{\odot}$ at $z \gtrsim 15$, inside of which early Population III stars form. The emergence of first star formation differs from halo to halo: the lower the halo mass, the later Population III stars begin to form. This is because a halo must reach a minimum mass required to host gas with a molecular hydrogen fraction of $f_{\mathrm{H}_{2}} \gtrsim 10^{-4}$, above which $\mathrm{H}_{2}$ cooling is efficient (e.g., Tegmark et al. 1997; Bromm et al. 2002). We see that Population III stars emerge at $z \lesssim 11$ in the less massive halos (HALO1, HALO2), while the more massive halos (HALO4, HALO5, HALO6) commence Population III star formation before $z=15$. The final virial masses at $z=0$ are less than $M_{\mathrm{vir}} \sim 1.6 \times 10^{9} M_{\odot}$ for three halos HALO1, HALO2, HALO3, while the others are slightly more massive with $M_{\mathrm{vir}} \sim 2.1 \times 10^{9}$ (HALO4), $M_{\mathrm{vir}} \sim 3.1 \times 10^{9} M_{\odot} \quad($ HALO5 $)$, and $M_{\mathrm{vir}} \sim 4 \times 10^{9} M_{\odot}$ (HALO6).

Note that early on, prior to reionization, star formation proceeds in an episodic fashion. This is a consequence of regulated star formation: the gas within the shallow potential well of a dwarf can easily be disrupted and evacuated by $\mathrm{SN}$ feedback, and it takes time for the halo gas to be replenished. This time delay gives rise to the episodic, bursty star formation with timescales of the order of approximately a few tens of megayears. As explained in Section 2, we gradually increase the external UV background, starting from $z=7$ to its full strength by $z=6$, indicated by the gray shaded vertical region in Figure 2; afterward, the background is present at full amplitude down to $z=0$.

The SFH of the simulated galaxies strongly depends on their halo mass. Quenching of star formation by reionization is clearly reflected in the noticeable drops in SFR during $z \sim 7-6$ in the less massive halos, HALO1, HALO2, and HALO3. Among them, HALO1 continues to form stars for about $\sim 30 \mathrm{Myr}$ beyond $z=7$, while HALO2 and HALO3 exhibit sharply truncated star formation as soon as the UV background is introduced. This residual star formation in HALO1 is due to the existence of dense gas, self-shielded from the UV background. Although two other halos, HALO4 and HALO5, also experience SFR quenching by the UV background, they exhibit a rebirth of star formation at late epochs, below $z=4$. Gas inflow in HALO5 is found to be driven by mergers with other halos at $z \sim 1.8$, such that the primary progenitor of HALO5 grows in mass by a factor of 5, leading to the ignition of star formation at $z \lesssim 0.5$. HALO4 shows late starbursts at $z \sim 3.9$ and $z \sim 0$ as well.

Interestingly, the most massive halo (HALO6) overcomes the effect of negative feedback from UV photoheating, since the halo already reaches a mass of $M_{\mathrm{vir}}=2.5 \times 10^{8} M_{\odot}$ at $z=7$, which is massive enough not to significantly be affected by external radiation. Therefore, stars can continuously form down to $z \sim 3$, followed by bursty star formation until $z=0$. These late-time bursts of star formation could be unlikely to occur if the simulated galaxy, HALO6, was accreted by the MW-like host at early times. As such, star formation would be suppressed by environmental factors. This implies that lowmass dwarfs $\left(M_{\mathrm{vir}} \gtrsim 2 \times 10^{9} M_{\odot}\right.$ at $\left.z=0\right)$ in the outskirts of the MW are likely to show signatures of late-time bursts of star formation. Or, such ongoing star formation and high stellar content $\left(M_{*} \sim 10^{6} M_{\odot}\right.$ at $\left.z=0\right)$ indicate that it is more reasonable to consider HALO6 as low-mass dwarf analogs such as Leo $\mathrm{P}$ or Leo $\mathrm{T}$ in the field rather than local UFDs. This is discussed further in the next section.

To explore the relative importance of $\mathrm{SN}$ feedback, compared to reionization, for the suppression of star formation, we perform a comparison simulation NOSN (see dotted lines in Figure 2). This run is identical to HALO1, except that the thermal SN feedback is excluded, but metals are ejected from stars to model the transition of star formation from Population III to Population II. We find that while reionization plays an important role in inhibiting star formation, $\mathrm{SN}$ feedback is crucial for the ultimate quenching of UFDs. Although the UV background is turned on in the same way as before (HALO1), no quenching of star formation is found this time, implying that reionization alone is insufficient to completely suppress star formation in the absence of SN feedback. We should note that we terminate the run NOSN at $z \sim 2$ for the sake of computational economy.

The bottom right panel of Figure 2 illustrates that all halos form more than $90 \%$ of stars prior to reionization, with the noticeable exception of HALO6. Following Ricotti \& Gnedin (2005), our five galaxies can be considered as "true fossils," as more than $70 \%$ of their stars formed before reionization. On the other hand, HALO6 only forms $\sim 30 \%$ of stars before 

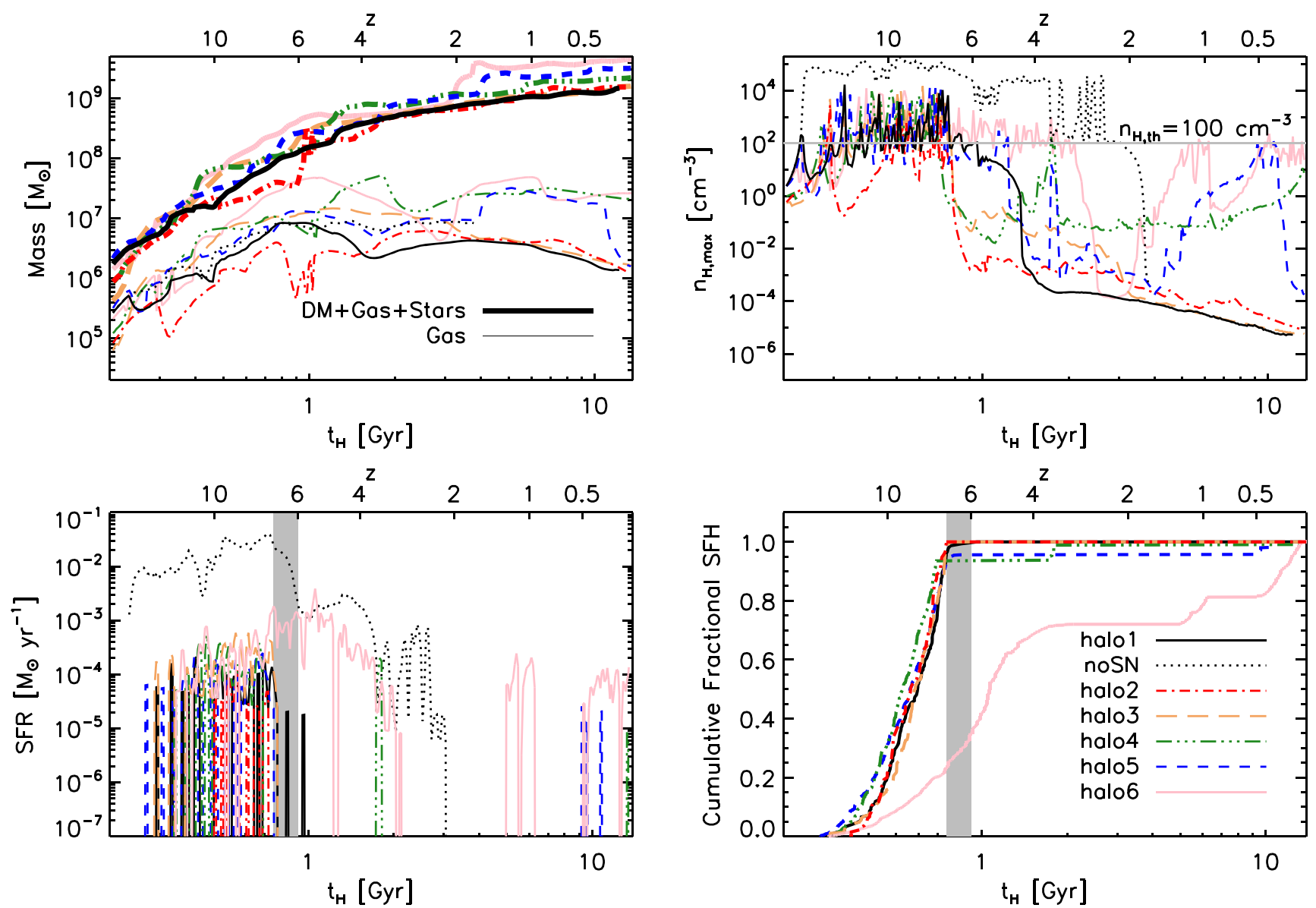

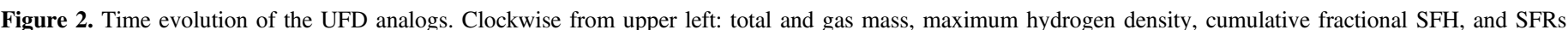

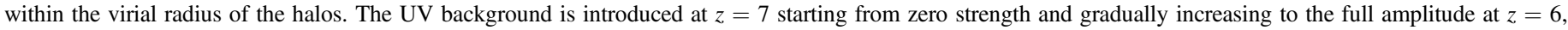

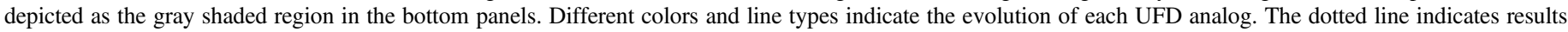

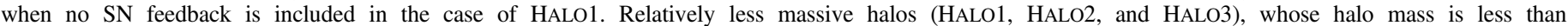

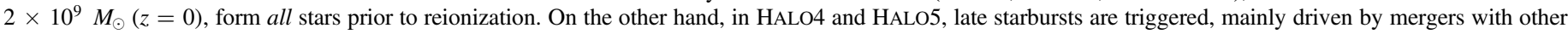

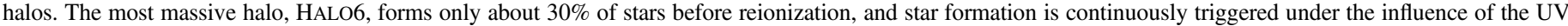

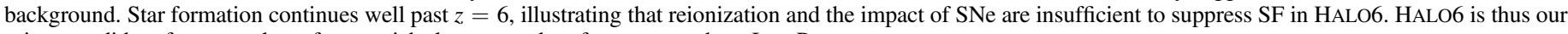
prime candidate for an analog of a gas-rich, low-mass dwarf system, such as Leo P.

reionization and continues to form stars down to $z=0$, and hence it could be categorized as a "polluted fossil."

\subsubsection{SFH of the Smaller Progenitor Halos}

Near the end of reionization, $z \sim 6$, the simulated halos, except HALO6, almost stop forming stars such that HALO1, HALO2, and HALO3 complete $100 \%$ of their star formation. Also, HALO4 and HALO5 already contain more than $92 \%$ of their stars by $z \sim 6$. Interestingly, we find that simulated analogs have experienced multiple mergers between halos, meaning that a substantial fraction of stars were formed in small progenitor halos $\left(M_{\mathrm{vir}} \lesssim 10^{8} M_{\odot}\right)$ at high redshift $(z \gtrsim 6)$ and assembled into a primary halo at later times. Figure 3 shows a fraction of stars in progenitor halos at $z=6$ that contain more than $5 \%$ of total stars found within a virial radius of the simulated analogs at $z=0$. The percentage indicates the fraction of stars formed at this time relative to the final stellar mass at $z=0$.

For instance, there were two massive progenitor halos of HALO1 at $z=6, M_{\text {halo }}=1.6 \times 10^{8} M_{\odot}$ and $M_{\text {halo }}=6 \times$ $10^{7} M_{\odot}$, containing $50 \%$ and $27 \%$ of stars, respectively. As the number of progenitors that contribute a non-negligible amount of stars increases, the fraction of stars formed in a primary halo, the most massive halo, declines as seen in HALO2, HALO4, and HALO5 of Figure 3. This means that the stellar mass growth of the simulated UFD analogs is a combination of both in situ star formation in a primary halo and stellar accretion via mergers of smaller progenitor halos.

The ability of the simulations to resolve small halos $\left(M_{\text {vir }} \lesssim 10^{8} M_{\odot}\right.$ at $\left.z \gtrsim 6\right)$ allows us to track down hierarchical structure formation to high redshifts, making it difficult to specify a "primary" halo, particularly for the relatively less massive analogs $\left(\mathrm{M}_{\mathrm{vir}}<2 \times 10^{9} M_{\odot}\right)$, in which progenitor halos were formed in low-density peaks. This confusion lessens for the relatively massive halo, i.e., HALO6 $\left(M_{\mathrm{vir}} \gtrsim 4 \times\right.$ $10^{9} M_{\odot}$ at $z=0$ ), as their progenitor halos grow rapidly by mergers and accretion since they originated from high-density peaks. As such, it is much easier for relatively massive halos to identify a primary halo. This is clearly seen in HALO6, where stars actively form in two progenitor halos before $z=6$, but afterward stars form in a single halo, leading to $81 \%$ of in situ stars in HALO6. On the other hand, unlike HALO6, the other five simulated halos almost complete star formation by $z \sim 6$-before stars were formed in multiple small progenitor halos. The fraction of stars formed in situ (within a primary halo) varies from halo to halo: $37 \%, 17 \%, 61 \%, 31 \%$, and $17 \%$ 


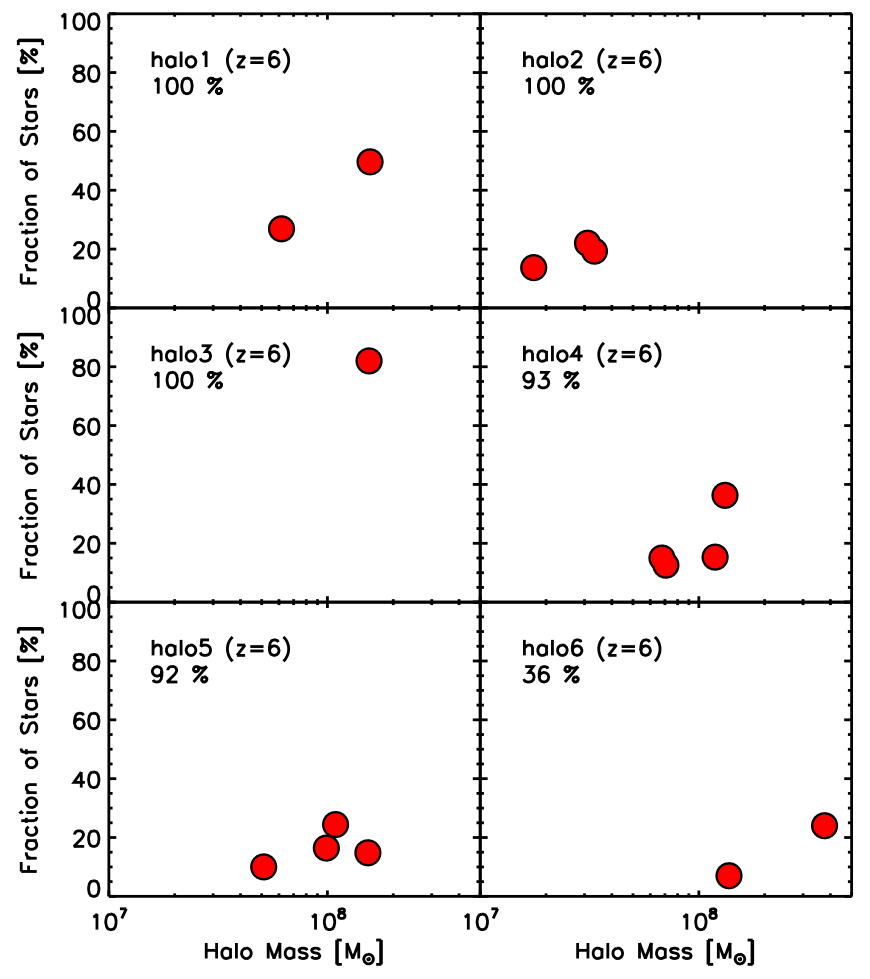

Figure 3. Fraction of stars within progenitor halos found at $z=6$ that contribute more than $5 \%$ of the total stars within the virial radius of the simulated galaxies at $z=0$. The percentage indicates the fraction of stars formed at this time relative to the final stellar mass at $z=0$. Prior to $z=6$, in all cases stars were formed in multiple different progenitors, not in a single primary halo. This means that a substantial amount of stars have originated from the hierarchical accretion of different halos, rather than being formed in situ. The ability of the simulations resolving small halos $\left(M_{\mathrm{vir}} \lesssim 10^{8} M_{\odot}\right)$ at $z>6$ allows us to track hierarchical structure formation at high-z. At early epochs $(z \gtrsim 6)$, star formation occurs simultaneously in multiple small halos $\left(M_{\mathrm{vir}} \lesssim 10^{8} M_{\odot}\right.$, at $\left.z>6\right)$, merging into a primary halo at later times. In all cases, except for the most massive HALO6, the halos almost stop forming stars at $z=6$. Prior to this point multiple star-forming small halos are present. Consequently, star formation of the simulated UFDs is found to be a combination of in situ star formation in a primary halo and a significant contribution from small progenitor halos that eventually merge into a primary halo. Note, however, that HALO6 continues to form stars until $z=0$, meaning that $81 \%$ of its stars are formed in situ within the virial radius of a single primary halo. This points to a notable distinction in the stellar mass buildup of UFDs vs. low-mass dwarfs.

from HALO1 to HALO5. This suggests that there is a notable distinction in the stellar mass buildup of UFDs versus low-mass dwarfs.

\subsection{Residual Gas: Halo6 as an Analog of a Low-mass, Gas- rich Dwarf}

Here we discuss whether any of our simulated halos are reasonable analogs to recently discovered low-mass, gas-rich dwarfs, such as Leo P, Leo T, Leo A, and DDO 210. We focus specifically on HALO6, which, being isolated and slightly more massive than the other dwarfs in our simulation suite, continues to form stars until $z=0$.

Whether or not a halo can form stars at a given time within the simulation can be illustrated by the maximum gas density achieved inside the virial radius of the simulated halos, shown in the top right panel of Figure 2. In this panel, we compare the maximum hydrogen number density with the adopted density threshold of $n_{\mathrm{H}}=100 \mathrm{~cm}^{-3}$ (solid horizontal line), above which gas can form stars. The maximum gas density can get as high as $n_{\mathrm{H}} \sim 10^{4} \mathrm{~cm}^{-3}$ at high redshift $z \gtrsim 7$. The final maximum gas density at $z=0$ is heavily dependent on the specific SFH experienced by a galaxy. For example, a majority of gas is evacuated by both the UV background and SN feedback in the low-mass halos (HALO1, HALO2, HALO3); hence, little gas has remained, eventually resulting in the very low maximum density of $n_{\mathrm{H}} \sim 10^{-5} \mathrm{~cm}^{-3}$ at $z=0$. Even though HALO5 is massive enough to form stars at late epochs, SN feedback from late starbursts significantly evacuates the gas from the halo, such that the maximum density is as low as $n_{\mathrm{H}} \sim 10^{-4} \mathrm{~cm}^{-3}$ at $z=0$. Meanwhile, we identify highdensity gas with $n_{\mathrm{H}} \gtrsim 10 \mathrm{~cm}^{-3}$ in HALO6 even at the end of the simulation.

The total residual gas mass of low-mass UFDs, both hot and cold gas, is roughly a few times $10^{6} M_{\odot}(z=0)$ (see Table 1$)$, but for HALO6 it can be as high as $2.6 \times 10^{7} M_{\odot}(z=0)$. We find that the ionized gas fraction at $z=0$, defined as gas with a free electron fraction of $f_{\text {elec }} \gtrsim 0.99$, is $\sim 54 \%$ in HALO6 and $\sim 74 \%$ in HALO4, whereas the gas is completely ionized in the other halos. Figure 4 illustrates the morphology of the refined region, centered on HALO4, at $z=7.5$ (top panels) and at $z=0$ (bottom panels). From left to right, each panel shows DM overdensity, hydrogen number density, gas temperature, and gas metallicity. At $z=7.5$, given the distribution of the heated and polluted gas by the SN explosions, stars are formed not only in the main halo, denoted as the white circle at the center of the top panels, but also out of gas in multiple progenitor halos that eventually have been assembled into the main halo at $z=0$. Owing to the ongoing star formation at $z=0$, the gas in HALO4 is significantly heated up to $T \gtrsim 10^{5}$ $\mathrm{K}$, as shown in the temperature map.

Interestingly, we find that a substantial amount of the residual gas of HALO6 at $z=0$ exists in a neutral phase. The mass of warm neutral gas, defined as $T<5000 \mathrm{~K}$ and $n_{\mathrm{H}}>0.5 \mathrm{~cm}^{-3}$, is $M_{\mathrm{H}} \sim 4.6 \times 10^{6} M_{\odot}(z=0)$, corresponding to $20 \%$ of the total gas. Also, there is a cold gas reservoir with $5.9 \times 10^{4} M_{\odot}$ $\left(T<1000 \mathrm{~K}\right.$ and $\left.n_{\mathrm{H}}>10 \mathrm{~cm}^{-3}\right)$, which serves as the gas supply for star formation. To date, neutral gas has not been detected in local UFDs $\left(M_{*}<10^{5} M_{\odot}, z=0\right)$, indicating that HALO6 is likely more representative of slightly more massive systems. In particular, the properties of HALO6 at $z=0$ are similar to those of gas-rich low-mass dwarfs like Leo $\mathrm{T}\left(M_{\mathrm{H} \mathrm{I}} \sim\right.$ $\left.2.8 \times 10^{5} M_{\odot}\right), \quad$ Leo $\mathrm{P} \quad\left(M_{\mathrm{H}} \sim 8 \times 10^{5} M_{\odot}\right), \quad$ DDO 210 $\left(M_{\mathrm{H} \mathrm{I}} \sim 4 \times 10^{6} M_{\odot}\right)$, or Leo A $\left(M_{\mathrm{H} \mathrm{I}} \sim 1.1 \times 10^{7} M_{\odot}\right)$ (see Table 2; McConnachie 2012; McQuinn et al. 2015).

This work could suggest that the observed gas-rich, lowmass dwarfs listed in Table 2 are explainable within a cosmological context if they have not been subjected to environmental effects (ram pressure stripping, etc.) for the bulk of their evolution. Therefore, the existence of dense gas, not seen in the UFDs within the virial radius of the MW, could be indirect evidence of the role of the environmental effects on quenching star formation (e.g., Wetzel et al. 2015). We find that HALO6 already forms $\sim 30 \%$ of stars at $z=6$, corresponding to stellar mass of $M_{*}=2.6 \times 10^{5} M_{\odot}(z=6)$. It indicates that even if HALO6 was quenched after reionization, it would not look like a UFD $\left(M_{*}<10^{5} M_{\odot}\right.$ at $\left.z=0\right)$, meaning that there likely is a hard upper limit on the UFD halo mass.

\subsection{Metal Enrichment}

Given that the simulated galaxies have experienced multiple mergers with other halos over cosmic time, it is expected that 

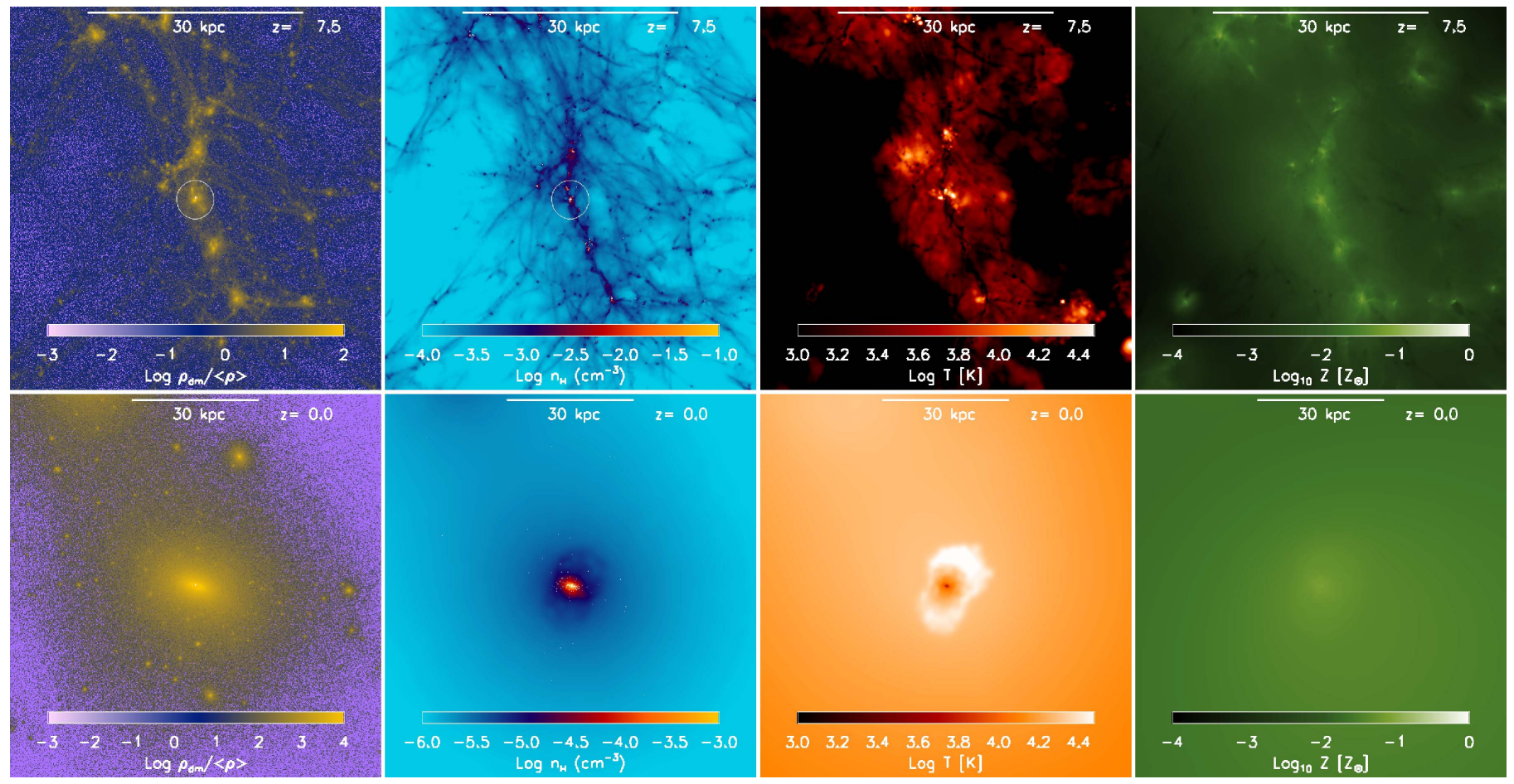

Figure 4. Visualization of the zoom-in region, centered on HALO4, at $z=7.5$ (top panels) and $z=0$ (bottom panels). From left to right: each panel displays DM overdensity relative to average density in universe, hydrogen number density, gas temperature, and gas metallicity. The radius of the primary halo, the most massive progenitor at a given time, is denoted as the white circle in the DM overdensity map (top left panel). At $z=0$, HALO4 still forms stars, some of which explode as SNe. The gas heated by the SN explosions is clearly shown in the temperature map at $z=0$. We see that, at $z=7.5$, stars are formed and explode as SNe within multiple progenitor halos and are then assembled into the primary halo. As a result, the galaxy at $z=0$ is composed of a significant fraction of stars that have originated in different halos that were later cannibalized by the primary.

Table 2

Summary of Gas-rich Nearby Low-mass Dwarfs

\begin{tabular}{lcc}
\hline \hline Dwarfs & $M_{*}\left(10^{5} M_{\odot}\right)$ & $M_{\mathrm{HI}}\left(10^{5} M_{\odot}\right)$ \\
\hline Leo A & 60 & 110 \\
DDO 210 & 16 & 41 \\
Leo P & 5.6 & 9.3 \\
Leo T & 1.4 & 2.8 \\
HALO6 & 8.8 & 44 (warm) $/ 0.59$ (cold) \\
\hline
\end{tabular}

Note. Column (1): names of the observed gas-rich low-mass dwarfs and HALO6. Column (2): stellar mass in $10^{5} M_{\odot}$. Column (3): neutral hydrogen mass in $10^{5} M_{\odot}$. The data are from McConnachie (2012) for Leo A, DDO 210, and Leo T and from McQuinn et al. (2015) for Leo P.

some fraction of stars could have formed in different halos and been accreted later onto the primary halo, i.e., the most massive progenitor at any given time. In order to distinguish the properties of stars formed in situ in a primary halo from those of stars formed externally and accreted at later epochs, in Figure 5 we compare the stellar metallicity as a function of formation time. In the top panels, we plot all stars inside the virial radius of HALO1 (left) and HALO6 (right) at $z=0$, regardless of their formation site. In the bottom panels we compare with stars formed only within the primary halo (in situ). Note that each symbol (downward-pointing triangle) indicates a single Population II stellar cluster.

We note that about $40 \%$ and $19 \%$ of stars in HALO1 and HALO6, respectively, at $z=0$ have originated from different

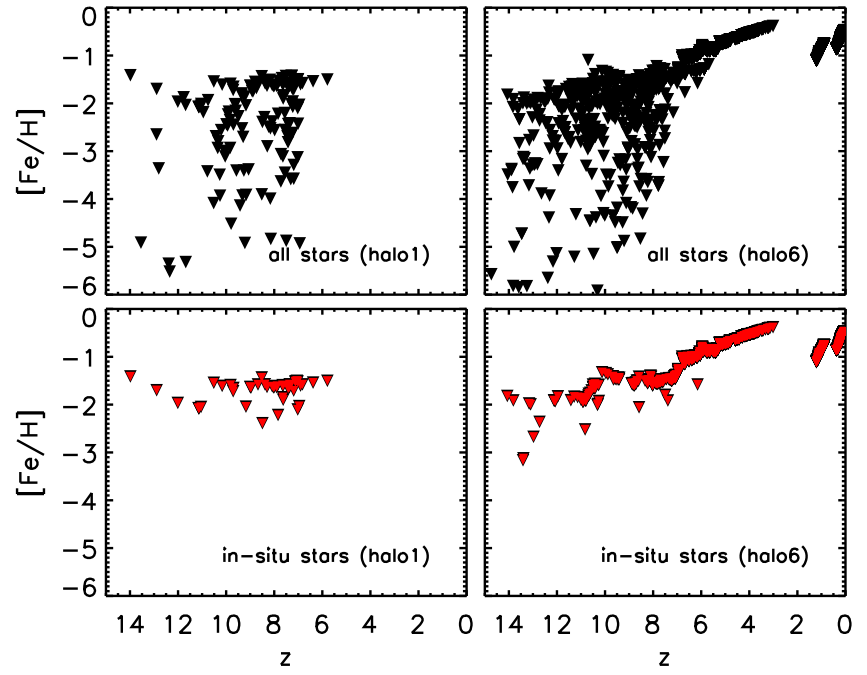

Figure 5. Externally vs. internally formed stars. Top panel: stellar metallicity vs. formation time for all stars located within the virial radius of a halo at $z=0$. Bottom panel: subset of stars formed in situ in the primary progenitor of a halo. The left and right panels are for HALO1 and HALO6, respectively. The absence of low-metallicity stars $([\mathrm{Fe} / \mathrm{H}] \lesssim-3)$ among the in situ sample indicates that low-metallicity stars have originated from different progenitor halos and were assembled into the primary halo at later epochs via mergers. Specifically, such low-metallicity stars are polluted via external enrichment, where metals originate in neighboring halos that host Population III SNe. These low-metallicity stars only form at early times $z \gtrsim 6$ when the cross-pollution is efficient owing to the proximity between halos. This finding is a general feature in all of the simulated analogs. 
progenitor halos and were assembled into the primary halo at later times via mergers. We can further classify these externally formed stars into two categories: (1) stars formed out of gas that is externally metal polluted owing to the proximity of neighboring halos hosting SNe, and (2) stars born within a halo that is independently massive enough to form Population III stars and subsequent generations of stars (in situ). The latter case is similar to a primary halo, which contains Population III stars and subsequently formed stars via self-enrichment. A striking difference evident in Figure 5 is how broadly stellar metallicity is distributed for all stars, whereas it is concentrated between $[\mathrm{Fe} / \mathrm{H}]=-2$ and $[\mathrm{Fe} / \mathrm{H}]=-3$ for the in situ sample, except for one stellar cluster with $[\mathrm{Fe} / \mathrm{H}]=-3.7$. The absence of low-metallicity stars $([\mathrm{Fe} / \mathrm{H}] \lesssim-3)$ among the in situ sample indicates that low-metallicity stars have preferentially come from different progenitor halos and were later acquired by the main halo through mergers.

This can be explained as follows. When a Population III star triggers an SN explosion, the surrounding gas is heated and evacuated from the host halo, locally suppressing further star formation for a certain time period. Detailed studies regarding the duration of this temporary lull in star formation in the wake of a Population III SN have suggested that it is of the order of a few times 10 Myr for a conventional SN energy of $10^{51} \mathrm{erg}$. Or, it lasts up to a few hundred megayears for a powerful PISN explosion with $10^{52} \mathrm{erg}$, within a host halo of $M_{\text {vir }} \sim 10^{5}-10^{6} M_{\odot}(z \gtrsim 10)$ (e.g., Greif et al. 2009; Ritter et al. 2012; Jeon et al. 2014).

During this period of inhibited star formation, metals are propagated into the ISM and IGM and can be mixed with pristine gas residing in neighboring minihalos. If the gas density in such polluted neighboring halos happens to be high enough to form stars, metal-enriched Population II stars would be born. Because only small amounts of metals would be delivered to neighboring halos, the metallicity of the gas that is located in the central region of the externally enriched halo would be low, giving rise to the formation of low-metallicity stars. Note that owing to the compactness of the early universe, the typical distance between minihalos with $M_{\mathrm{vir}} \sim 10^{5} M_{\odot}(z \gtrsim 10)$ is $\sim 300-500$ pc, making it possible to transfer metals to neighboring halos (Smith et al. 2015).

The bottom left panel of Figure 5, on the other hand, implies that the in situ stars are likely born in an environment that is internally polluted, thus experiencing self-enrichment. The primary progenitor halo forms the first Population III stars at $z \sim 14$, and the following Population III SN explosion leads to a suppression of star formation for $\sim 50 \mathrm{Myr}$. Afterward, the ejected gas is replenished along the cosmic web, and the central gas again reaches densities high enough to form Population II stars. We find that in a given halo, only one or two Population II clusters are formed out of gas directly polluted with metals from Population III SNe, implying that only a few Population II stars will preserve the intrinsic chemical signatures of Population III stars (Ji et al. 2015). While HALO1 stops forming stars at $z \sim 5.5$, HALO6 continues to form stars down to $z=0$, steadily self-enriching the gas within the primary progenitor halo. As shown in the top panels of Figure 5, lowmetallicity stars $\left([\mathrm{Fe} / \mathrm{H}] \lesssim 10^{-3} \mathrm{Z}_{\odot}\right)$ tend to be generated at early times before $z=6$ when the cross-metal pollution is efficient owing to the proximity between low-mass halos with $M_{\text {vir }}<10^{8} M_{\odot}$.

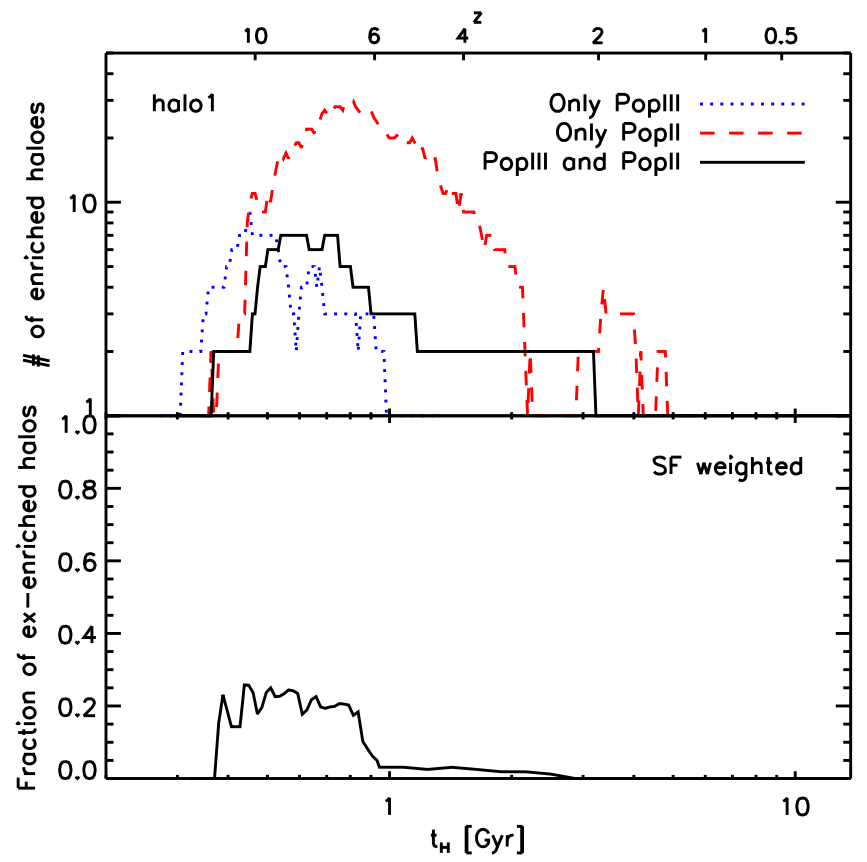

Figure 6. Top panel: number of halos that are eventually incorporated into HALO1, divided according to their metal enrichment path. The blue dotted line shows the number of halos that only host Population III stars or their remnants, while the red dashed line depicts halos with only Population II stars. Finally, the black solid line indicates halos that host both Population III and Population II star formation. The substantial number of Population-II-only halos (see red dashed line) implies that a large fraction of halos are externally polluted with metals from neighboring halos that host SNe. Bottom panel: fraction of externally enriched halos weighted by star formation in each halo. As stated above, even though there are a number of externally polluted halos, they tend to host only one or multiple star clusters, making a small percentage contribution to the total star formation. Therefore, effectively $\sim 20 \%$ of star-forming halos at $\gtrsim 6$ were polluted via external metal enrichment. These trends are shown in all of the simulated analogs.

In order to estimate how many externally enriched progenitors contribute to the formation of the simulated dwarf galaxy at $z=0$, we present in Figure 6 the number of halos with different metal enrichment paths that end up in the primary halo (HALO1) through mergers. The red dashed line indicates the number of halos that host only Population II stars without the presence of any Population III stars or their remnants, implying that they have been externally polluted. The blue dotted and black solid lines correspond to halos hosting Population III only, or both Population III and Population II stars, respectively. The displacement between the blue dotted and black solid lines can be understood as the time delay when Population III star formation transitions to Population II, in halos eventually hosting Population III remnants and Population II stars. This time delay turns out to be about a few tens of megayears.

Interestingly, the number of externally enriched halos increases up to $\sim 30$ until $z \sim 6$, as the ISM and IGM are enriched with metals from Population III and Population II $\mathrm{SNe}$, but their number declines after reionization, mainly due to the inability to form stars in low-mass halos from gas heated by reionization. We point out that as global metal enrichment progresses, no Population-III-only halos exists $\sim 1$ Gyr after the big bang. Even though there are a number of externally polluted halos prior to $z=6$, they only host a single or 


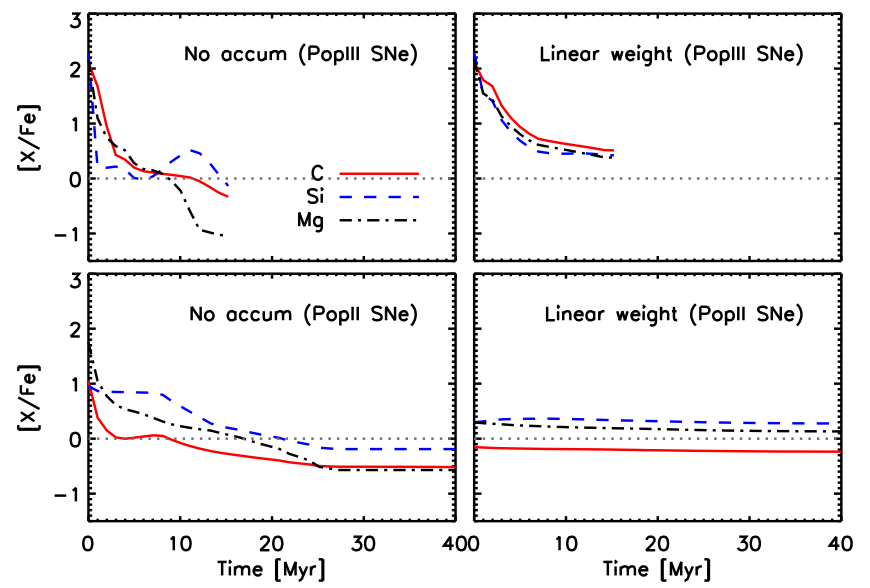

Figure 7. Evolution of metal yields of Type II SNe from Population III (top panels) and Population II stars (bottom panels). We assume two cases: (1) metals from individual SN events immediately propagate onward and thus do not accumulate in the high-density region at the center of a halo (left), or (2) their transport is slow, such that metals pile up in the central region, giving rise to an effective yield (right). For the former case, subsequently forming stars are sampling the metal yields from the most recent SN only, whereas the latter case means that next-generation stars will be born out of gas polluted with metals from multiple SNe. Note that Population III yields are displayed only up to $\sim 15 \mathrm{Myr}$, corresponding to the lifetime of a $10 M_{\odot}$. Population III star, the lowest mass considered in this work. The Population II yields extend to longer times, in accordance with their lower masses.

multiple star clusters, providing the small contribution to overall star formation. In order to estimate the effective fraction of externally polluted halos, in the bottom panel of Figure 6 we present the fraction of externally enriched halos weighted by the number of stars within each halos. About $20 \%$ of halos experience the cross-metal pollution between $z \sim 11$ and $z \sim 6$ in HALO1. Note that all these findings are general features in all of the simulated analogs.

\subsection{Chemical Abundances}

In this section, we first explain the general trends of the resultant chemical abundances, depending on different stellar populations, Population III and Population II. Then, we discuss individual abundances, carbon and alpha elements based on the expected trends, and finally present MDFs and comparisons to results for observed UFDs.

\subsubsection{General Trends}

To better understand our simulation results, we now introduce a simple toy model for the chemical evolution from multiple SNe. Specifically, in Figure 7 we illustrate the evolution of metal yields, as a function of time since the most massive star has exploded, for a Population III (top) and Population II (bottom) cluster. The panels on the left and right explore different assumptions for the efficiency of metal transport (see below). We only present the Population III yields up to $15 \mathrm{Myr}$, which corresponds to the lifetime of the lowest-mass, and thus longest-lived, Population III star for our chosen IMF, where $m_{* \text {, Pop III }}=10 M_{\odot}$ (Schaerer 2002). For Population II, we extend the yields to later times, corresponding to the lower mass progenitor stars present in this case $\left(m_{*, \text { Pop II }}=8 M_{\odot}\right)$. In our idealized enrichment model, metals are released in time such that those from the most massive stars are ejected first. Therefore, the gas metallicity at the SN explosion site changes over time, and one of the key factors is how rapidly metals can be transported to large distances. For example, if the timescale for metal transport is shorter than the time between consecutive $\mathrm{SNe}$, then the metallicity of a newly formed star will be determined mostly by SN yields from the most recent event. In this case, metals from earlier $\mathrm{SNe}$ would already have escaped into the surroundings. Conversely, if their propagating timescale is long, the metals ejected from different SNe will linger in the central region of a halo. The metallicity of a newly formed star would then reflect averaged yields, deposited by multiple SNe.

To further construct our simple model, we consider two extreme cases of how efficiently metals are transported: (1) ejected metals are not accumulated in the central star-forming region and are instead immediately dispersed into the IGM, or (2) a certain fraction of the metals from each SN remains in the central region, thus giving rise to effective yields. The latter are calculated with a linear weight, measuring the time since explosion, such that the most recent SNe contribute most to the average. Or formally, we employ a mass-dependent weight as follows: $w\left(m_{*}\right)=\left(t-\Delta t\left(m_{*}\right)\right) / t$, where $t$ is the time since the explosion of the most massive star in the cluster and $\Delta t\left(m_{*}\right)$ is the time since a progenitor with mass $m_{*}$ exploded. We find that the general trend of the yields from Population III and Population II SNe is substantially different. First, Population III SNe produce more carbon compared to Population II events. Second, for Population III yields, the $[\mathrm{C} / \mathrm{Fe}]$ ratio is similar to or larger than $[\alpha / \mathrm{Fe}]$, especially $[\mathrm{Mg} / \mathrm{Fe}]$, while these trends are opposite for Population II yields. These distinctive yield trends thus allow us to distinguish whether stars are formed under the dominant influence of Population III or Population II SNe.

We now compare these trends, obtained within the toy model above, with the detailed enrichment results from our full simulations. As discussed above, in situ stars likely form in environments internally enriched by Population II SNe, while stars formed through external metal enrichment are likely to show Population III signatures. These trends are evident in Figure 8 , where we plot the chemical abundances of stars, formed in our simulations, as a function of their formation time (left) and their metallicity (right). Recall that individual, downward-pointing triangles indicate single Population II clusters. Except for the very first stellar cluster, the majority of in situ stars (bottom panels) formed under the influence of Population II SNe, given that $[\mathrm{Si} / \mathrm{Fe}]$ is larger than $[\mathrm{C} / \mathrm{Fe}]$, which is the predicted trend for Population II SNe. As an exception, the very first stellar cluster was born out of gas enriched by Population III SNe, indicated by similar $[\mathrm{C} / \mathrm{Fe}]$ and $[\mathrm{Si} / \mathrm{Fe}]$ ratios, consistent with Population III SN yields. The chemical abundances of all stars, shown in the top panels of Figure 8, are established by a mixture of two systems: (1) halos, mainly polluted via internal enrichment, hosting both Population III and Population II stars; and (2) externally contaminated halos. The existence of stellar clusters that exhibit comparable $[\mathrm{C} / \mathrm{Fe}]$ and $[\mathrm{Si} / \mathrm{Fe}]$ ratios implies that their stars are likely formed out of gas mostly enriched by Population III stars. To the contrary, a lower value of $[\mathrm{C} / \mathrm{Fe}]$ relative to $[\mathrm{Si} / \mathrm{Fe}]$ signals the dominant contribution from Population II SNe.

\subsubsection{Carbon}

Figure 9 shows select chemical abundances of Population II stars within the virial volume of our simulated galaxies at 

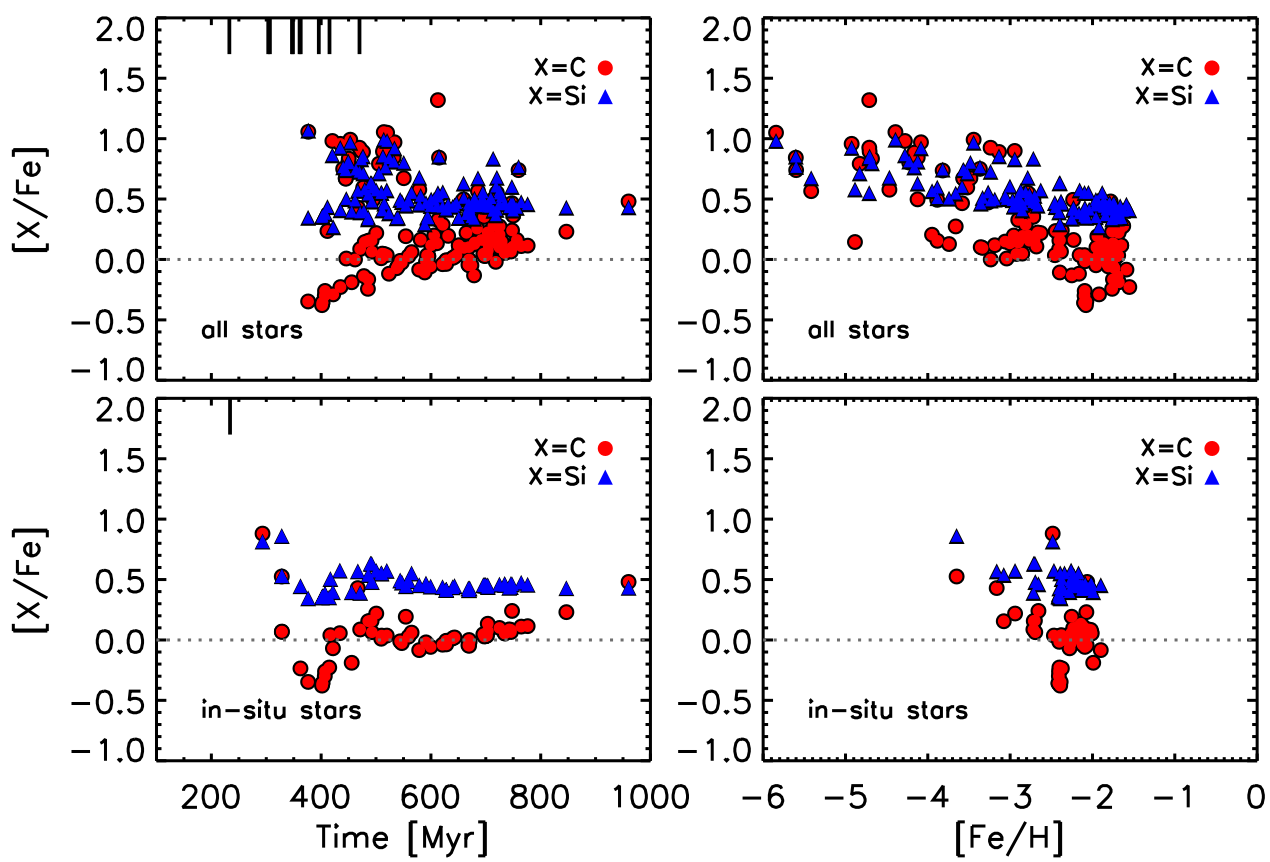

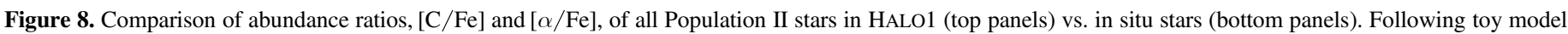

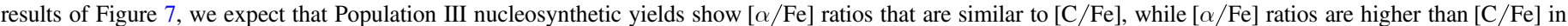

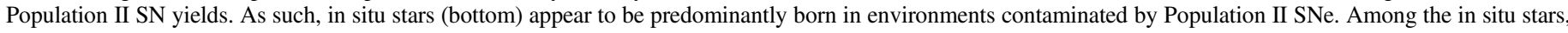

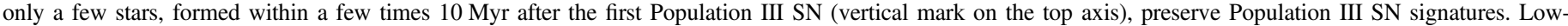

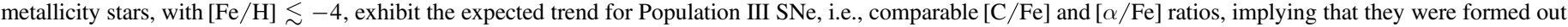
of gas enriched by Population III stars.

$z=0$, specifically $[\mathrm{C} / \mathrm{Fe}]-[\mathrm{Fe} / \mathrm{H}]$ and $[\alpha / \mathrm{Fe}]-[\mathrm{Fe} / \mathrm{H}]$ (left and right columns). The color scheme denotes the formation time of stars, where grayish colors correspond to times before $t_{\mathrm{H}} \sim 4 \mathrm{Gyr}$ and reddish ones to more recent epochs. As we have discussed in the previous sections, star formation in the three halos, HALO1, HALO2, and HALO3, is truncated close to reionization, and thus all their stars form before $1 \mathrm{Gyr}$, shown in gray scale. The stars in the other three halos that experience late bursts or continuous star formation span a much wider range of formation times, reflected in the presence of squares with reddish colors.

High- and medium-resolution spectroscopic studies of individual member stars in UFDs have revealed that the fraction of carbon-enhanced metal-poor (CEMP) stars increases with decreasing metallicities (see Beers \& Christlieb 2005, for details; see also, e.g., Aoki et al. 2007; Norris et al. 2013; Yong et al. 2013). Usually, CEMP stars are defined as metal-poor stars with carbon-to-iron ratios above $[\mathrm{C} / \mathrm{Fe}]=0.7$ at $[\mathrm{Fe} / \mathrm{H}] \lesssim-2$ (e.g., Aoki et al. 2007). Several possible mechanisms for their origin have been suggested, including (i) rapidly rotating massive Population III stars $\left(m_{*}=\right.$ 40-120 $M_{\odot}$ ), capable of releasing large amounts of CNOenhanced material (e.g., Meynet et al. 2006); (ii) binary systems with a companion star that undergoes an AGB phase, thus transferring carbon onto a low-mass, long-lived, metalpoor star; or (iii) Population III SNe $\left(m_{*}=1-40 M_{\odot}\right)$ with a low explosion energy. In the last case, iron-peak elements are more likely to be locked up in the emerging central remnant, whereas those with lower atomic number, such as carbon, are easily ejected. In addition, such ejecta are more likely to rapidly fall back since low SN energies are inefficient to drive the gas out of a host halo (e.g., Umeda \& Nomoto 2003; Iwamoto et al. 2005; Cooke \& Madau 2014; Salvadori et al. 2015).
Our simulation results indeed exhibit the presence of CEMP stars at low metallicities. Since we exclude the existence of binary systems, the occurrence of CEMP stars in this work is a consequence of Population III SNe that intrinsically produce abundant carbon (see Figure 7). Also, we find that the fraction of CEMP stars is higher at low metallicities, $[\mathrm{Fe} / \mathrm{H}] \lesssim-4$, such that this fraction declines from $>45 \%$ at $[\mathrm{Fe} / \mathrm{H}] \lesssim-4$ to $<20 \%$ at $[\mathrm{Fe} / \mathrm{H}] \lesssim-2$ for all halos. As demonstrated in the right panels of Figure 8 , low-metallicity $([\mathrm{Fe} / \mathrm{H}] \lesssim-3)$ stars with high carbon ratios of $[\mathrm{C} / \mathrm{Fe}]>0.7$ originate from Population III $\mathrm{SNe}$, characterized by their large intrinsic carbon production. As can also be seen, the $[\mathrm{C} / \mathrm{Fe}]$ ratio decreases toward near-solar values, giving rise to $\mathrm{C}$-normal stars, with increasing metallicity. This behavior is expected as a consequence of self-enrichment by Population II stars, where carbon yields are normal and the overall gas metallicity rises in the process.

We now compare our simulated abundances with the empirical record in select UFDs. For instance, the member stars of Segue 1 roughly trace the trend of an increasing number of CEMP stars toward lower metallicity, except for one star with $[\mathrm{C} / \mathrm{Fe}]=1.4$ at $[\mathrm{Fe} / \mathrm{H}]=-1.6$. Based on its enhanced abundance of neutron-capture elements, Frebel et al. (2014) suggest that this outlier star might be associated with a binary system where carbon is transferred from a companion. On the other hand, high-resolution spectroscopy shows that stars in Bootes I include both CEMP signatures at low metallicities $([\mathrm{Fe} / \mathrm{H}]<-3.0)$ and $\mathrm{C}$-normal ones at $[\mathrm{Fe} / \mathrm{H}] \gtrsim-3$ (e.g., Gilmore et al. 2013; Ishigaki et al. 2014; Frebel et al. 2016). Given the coexistence of CEMP and C-normal stars, Bootes I thus appears to be very similar to our simulated galaxies, in particular HALO3, where both classes of stars are naturally produced through a combination of Population III SNe and self-enrichment by Population II stars. 

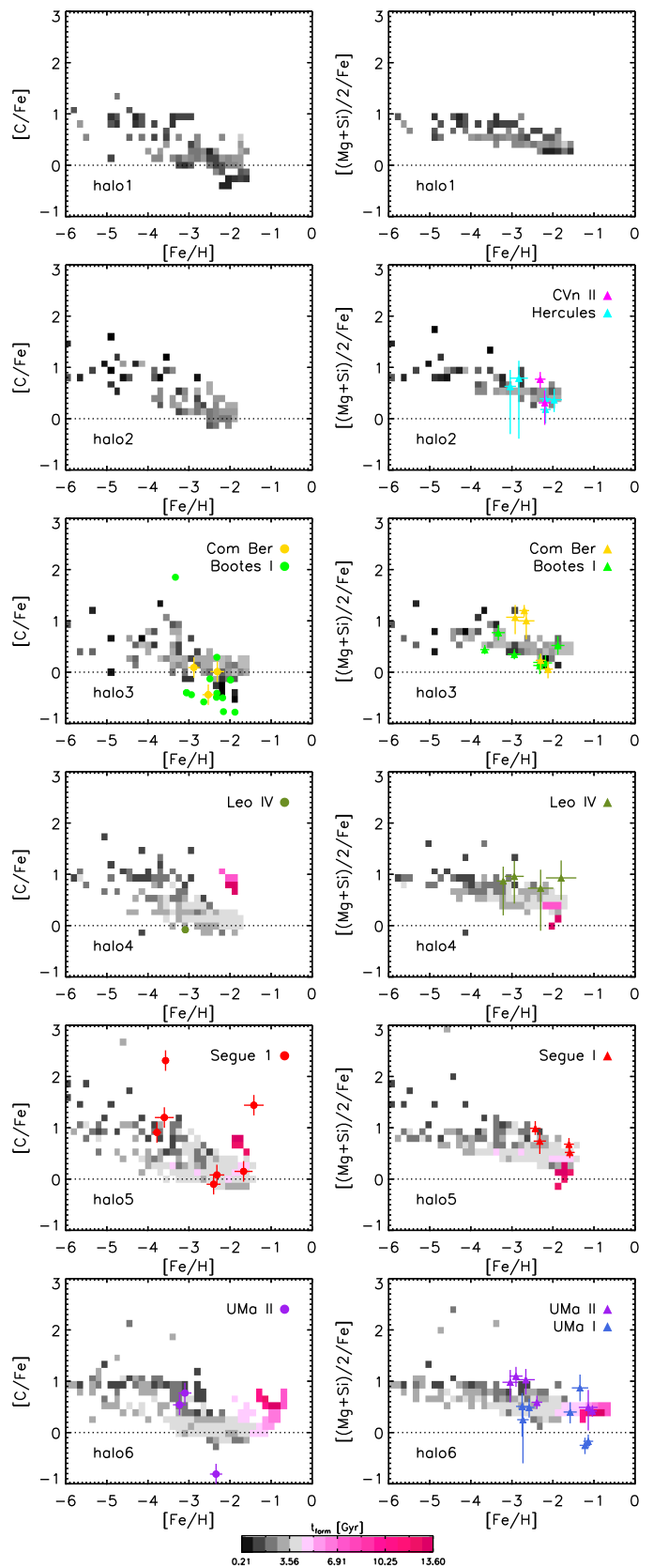

Figure 9. Stellar abundances in the simulated galaxies at $z=0$. Left column: $[\mathrm{C} / \mathrm{Fe}]$ vs. $[\mathrm{Fe} / \mathrm{H}]$. Right column: $[\alpha / \mathrm{Fe}]$ vs. $[\mathrm{Fe} / \mathrm{H}]$. The squares denote simulated abundances, with color indicating the formation time of stars, such that gray scale marks stars formed before $t_{\mathrm{H}} \sim 4 \mathrm{Gyr}$ and reddish colors those formed after $t_{\mathrm{H}} \sim 4 \mathrm{Gyr}$. The existence of CEMP stars, particularly at low metallicities, $[\mathrm{Fe} / \mathrm{H}] \lesssim-3$, is a consequence of Population III SNe that intrinsically produce large amounts of carbon. The enhanced $\alpha$-elements and the absence of clear knees, at which $[\alpha / \mathrm{Fe}]$ ratios decline, indicate that the gas in the galaxies is preferentially metal-enriched by Type II SNe, rather than Type Ia, from Population III and massive Population II stars. In the most massive HALO6 (bottom panels), the gas is metal enriched up to $[\mathrm{Fe} / \mathrm{H}] \sim-0.6$, owing to its extended $\mathrm{SFH}$. Observational data of stellar abundances in select LG UFDs are displayed with the symbols, as labeled in the legends. For carbon, the data are taken from Simon et al. (2010) for Leo I, Frebel et al. (2010) for UMa II and Com Ber, and Frebel et al. (2014) for Segue 1. For Bootes I, the data are from Frebel et al. (2016), who provide a list of all high-resolution measurements from the literature. For the alpha elements, we use the data given by Frebel et al. (2010) and Gilmore et al. (2013). A detailed comparison between the simulated UFD analogs and the observed UFDs is discussed in Section 3.5.

\subsubsection{Alpha Elements}

The run of $[\alpha / \mathrm{Fe}]$ over $[\mathrm{Fe} / \mathrm{H}]$ serves as a well-known cosmic clock, encoding the timescale over which stars have formed. In particular, the existence of a knee, where $[\alpha / \mathrm{Fe}]$ begins to decline, has been suggested as a reflection of a long duration of star formation. Given sufficient time, Type Ia SNe would begin to contribute, producing more iron compared to alpha elements. As is evident in the right panels of Figure 9, we see roughly flat $[\alpha / \mathrm{Fe}]$ abundances at low metallicities, $[\mathrm{Fe} / \mathrm{H}] \lesssim-3$, in all runs. As discussed in the previous sections, such low-metallicity stars are likely to be formed via external metal enrichment by Population III SNe. We note that relatively massive halos, HALO4, HALO5, and HALO6, show considerable scatter in the $\alpha$-abundance ratio, including values as high as $[\alpha / \mathrm{Fe}] \gtrsim 2$. This scatter can be understood as follows. The number of progenitor halos, hosting Population III stars, rises with increasing halo mass. As a result, the gas is incorporated from multiple halos that are contaminated by different Population III SNe at a given time, leading to a large spread in $[\alpha / \mathrm{Fe}]$ ratio (see Figure 7). We should mention that our simulations can only trace a few alpha elements, including $\mathrm{Si}$ and $\mathrm{Mg}$, limiting our ability to compare to observations.

Among the simulations, the less massive halos, HALO1, HALO2, and HALO3, exhibit enhanced $[\alpha / \mathrm{Fe}]$ ratios over the entire metallicity range, extending to $[\mathrm{Fe} / \mathrm{H}] \sim-2$, without an obvious decline at high $[\mathrm{Fe} / \mathrm{H}]$. The absence of a distinct knee implies that the stars in these halos have formed out of gas that was preferentially polluted with metals from Type II rather than Type Ia SNe. In contrast, the most massive systems, HALO5 and HALO6, show hints of Type Ia SNe. For instance, stars formed late in HALO5 at $z \sim 0.5$ (see Figure 2) have nearor supersolar $[\alpha / \mathrm{Fe}]$ ratios at $[\mathrm{Fe} / \mathrm{H}] \sim-1.6$. Unlike the other halos, owing to the extended star formation, stellar metallicity in HALO6 increases, extending to $[\mathrm{Fe} / \mathrm{H}] \sim-0.6$. It should be noted that the $[\alpha / \mathrm{Fe}]$ ratio at $[\mathrm{Fe} / \mathrm{H}] \gtrsim-2$ is below that at lower metallicities, but $[\alpha / \mathrm{Fe}]$ still remains supersolar. This is due to the continuous star formation, followed by Type II SNe, which are likely to wash out the impact of Type Ia SNe, by continuously producing abundant alpha elements.

As a limiting case, Frebel \& Bromm (2012) proposed a "oneshot enrichment" scenario, where feedback from massive Population II stars, themselves showing pure Population III chemical signatures, evacuated the remaining gas from their host halo, thus completely suppressing further star formation until $z=0$. Thus, any long-lived Population II stars in these systems would exhibit a constant alpha-element ratio, $[\alpha / \mathrm{Fe}]=0.35$, which is solely determined by the yields of Population III Type II SNe. This should, however, be considered an extreme case, valid for small, isolated halos not undergoing continued accretion or mergers. Thus, our simulated galaxies are not the case in which a halo grows in mass, making it harder for the gas in the halo with a deepened potential well to escape, rendering the effectiveness of stellar feedback weaker. Furthermore, the evacuated gas will begin to fall back within the order of a few times $10 \mathrm{Myr}$, triggering subsequent episodes of star formation (e.g., Ritter et al. 2012; Jeon et al. 2014). This multigenerational star formation is reflected as scatter in the $[\alpha / \mathrm{Fe}]$ ratios of observed LG dwarfs.

Measurements of $\alpha$-elements in MW UFDs are provided by several studies, including Vargas et al. (2013), where individual 


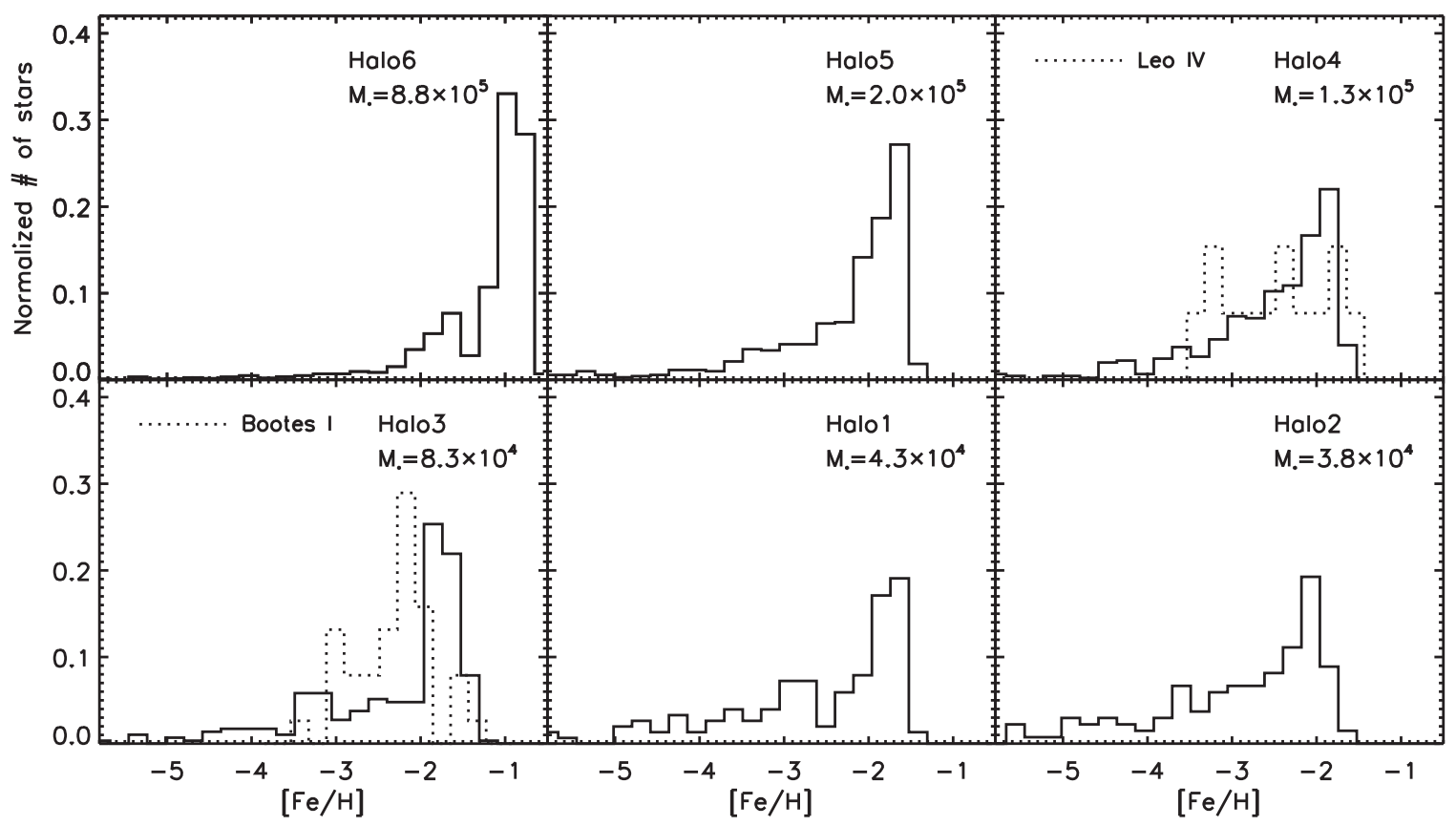

Figure 10. Normalized MDF of Population II stars for each halo, ordered according to decreasing stellar mass (beginning in the top left panel). The MDF of halos with stellar mass larger than $M_{*}>10^{4} M_{\odot}(z=0)$ shows a peak at $[\mathrm{Fe} / \mathrm{H}] \sim-2$, below which it gradually declines toward the low-metallicity regime. Such MDF shape is a natural consequence of both Population III and Population II SNe contributing: the peak at [Fe/H] $\sim-2$ is due to the continuing self-enrichment by Population II stars, whereas the low-metallicity tails represent stars formed in neighboring halos under the influence of Population III SNe. As a result, such low-metallicity stars are likely to preserve the signatures of Population III SNe. Owing to the prolonged SFH of HALO6, the MDF peaks at [Fe/H] $\sim-1$, but none of the observed UFDs can explain such distribution. As explained, it is reasonable for HALO6 to be classified as low-mass dwarfs rather than UFDs. An alternatively possible explanation would be that environmental effects within the virial radius of the MW could play a critical role in terminating star formation in such systems.

alpha-abundance ratios are presented for 61 red giant branch stars in eight UFDs. They found that all UFDs are likely to show high $[\alpha / \mathrm{Fe}]$ ratios at low $[\mathrm{Fe} / \mathrm{H}]<-2.5$, which is consistent with the results of our simulations. In particular, Segue 1 and UMa II exhibit enhanced alpha abundances across the observed metallicity range $-3.5<[\mathrm{Fe} / \mathrm{H}]<-1.0$, indicative of the signature of Type II SNe, while the other six systems show a general trend of decreasing $[\alpha / \mathrm{Fe}]$ ratio with increasing $[\mathrm{Fe} / \mathrm{H}]$. However, none of the observed UFDs have yet revealed $[\alpha / \mathrm{Fe}]$ ratios below $[\mathrm{Fe} / \mathrm{H}] \sim-3.5$, preventing us from comparing our predictions on extremely low metallicity stars with observations.

Overall, our predictions for the $[\alpha / \mathrm{Fe}]$ ratio show good agreement with observations over the metallicity range $-3.5<[\mathrm{Fe} / \mathrm{H}]<-1.0$. The observations also show that most UFDs exhibit no clear knee, favoring the interpretation that their stars were born out of gas dominantly polluted by Type II SNe (e.g., Gilmore et al. 2013; Vargas et al. 2013). To be specific, the measured $[\alpha / \mathrm{Fe}]$ ratios of $\mathrm{CVn}$ II and Hercules are consistent with those of HALO2, while the values of Coma Berenices and Bootes I agree with those of HALO3. These are relatively less massive halos, $M_{\text {vir }} \lesssim 3 \times 10^{9} M_{\odot}(z=0)$, experiencing truncated star formation. Furthermore, HALO4 and HALO5 show excellent agreement with Leo IV and Segue 1 , in combination with their measured $[\mathrm{C} / \mathrm{Fe}]$ ratios and the observed maximum metallicity.

\subsubsection{Metallicity Distribution Function}

In Figure 10, we display normalized stellar MDFs for the simulated galaxies, i.e., the fraction of Population II stars as a function of $[\mathrm{Fe} / \mathrm{H}]$, in order of decreasing stellar mass, beginning from the top left panel. The general shape of the
MDF exhibits a peak that gradually declines toward the lowmetallicity regime and sharply drops above the peak. The location of the peak shifts from $[\mathrm{Fe} / \mathrm{H}] \sim-1$ for the most massive halo, HALO6, with a stellar mass of $M_{*} \sim 8.8 \times 10^{5} M_{\odot}(z=0)$, to $[\mathrm{Fe} / \mathrm{H}] \sim-2$ for HALO2, where $M_{*} \sim 3.8 \times 10^{4} M_{\odot}(z=0)$. Our derived MDFs reflect the combined action of external metal enrichment by Population III stars and self-enrichment by Population II stars. Since the low-metallicity stars have predominantly originated in halos affected by Population III SNe, the resultant fraction is expected to be small owing to the brief Population III era, thus giving rise to the extended tails. The self-enrichment by Population II stars, on the other hand, increases the gas metallicity in their host halo, imprinting the peak at high $[\mathrm{Fe} / \mathrm{H}]$.

We also plot observed MDFs for select UFDs, shown as dotted lines, provided by Brown et al. (2014). Our simulations can approximately reproduce the range of metallicity, especially the upper limit, for Leo IV and Bootes I, but fail to simultaneously account for the peak of the observed MDFs. We find that none of the MDFs of the observed UFDs are matched with the MDF of HALO6 that reflects the prolonged star formation down to $z=0$, resulting in the peak at a relatively high metallicity $([\mathrm{Fe} / \mathrm{H}] \gtrsim-1)$ via self-enrichment. This discrepancy supports the assertion that the environmental effects within the virial radius of the MW are responsible for the truncated star formation in the relatively massive UFDs at late time, providing a constraint on the accretion epoch of UFDs. However, we should emphasize that the empirical record, in particular for low-metallicity stars, is currently still very much subject to small number statistics, such that definitive conclusions cannot be reached yet. 
Table 3

Summary of Comparison between the Simulated Halos and the Abundances of Observed UFDs

\begin{tabular}{lccc}
\hline \hline UFDs & $M_{*}\left[M_{\odot}\right]$ & Analogs & Similar Properties \\
\hline Segue I & $1.0 \times 10^{3}$ & HALO5 & {$[\alpha / \mathrm{Fe}],[\mathrm{C} / \mathrm{Fe}],[\mathrm{Fe} / \mathrm{H}]_{\max }$} \\
Com Ber & $3.7 \times 10^{3}$ & HALO3 & {$[\alpha / \mathrm{Fe}],[\mathrm{C} / \mathrm{Fe}]$} \\
UMa II & $4.1 \times 10^{3}$ & HALO6 & {$[\alpha / \mathrm{Fe}],[\mathrm{Fe} / \mathrm{H}]_{\max }$} \\
CVn II & $7.9 \times 10^{3}$ & HALO2 & {$[\alpha / \mathrm{Fe}]$} \\
Leo IV & $1.0 \times 10^{4}$ & HALO4 & {$[\alpha / \mathrm{Fe}],[\mathrm{C} / \mathrm{Fe}],[\mathrm{Fe} / \mathrm{H}]_{\max }$} \\
UMa I & $1.4 \times 10^{4}$ & NONE & $\cdots$ \\
Bootes I & $2.9 \times 10^{4}$ & HALO3 & {$[\alpha / \mathrm{Fe}],[\mathrm{C} / \mathrm{Fe}],[\mathrm{Fe} / \mathrm{H}]_{\max }$} \\
Hercules & $3.7 \times 10^{4}$ & HALO2 & {$[\alpha / \mathrm{Fe}],[\mathrm{C} / \mathrm{Fe}],[\mathrm{Fe} / \mathrm{H}]_{\max }$} \\
\hline
\end{tabular}

Note. Column (1): names of the observed UFDs. Column (2): stellar mass of the observed UFDs in $M_{\odot}$. Column (3): name of the most similar analogs among the simulated galaxies based on the chemical properties listed in column (4).

\subsection{Connection to the Observed UFDs}

In this section, we attempt to provide insight into the quenching and accretion timescales of the observed UFDs around the MW based on the similarities of their chemical abundances to our simulated halos. To be specific, we match the simulated UFD analogs with the observed LG UFDs by comparing the chemical properties, such as $[\alpha / \mathrm{Fe}],[\mathrm{C} / \mathrm{Fe}]$, and $[\mathrm{Fe} / \mathrm{H}]_{\max }$, which is the maximum metallicity. A summary of this comparison is listed in Table 3. We note that a comprehensive comparison should include other properties, such as the full SFH and gas content of each dwarf. However, we focus here on the impact of the new physics we have introduced, which manifests in the chemical abundances of the simulated halos. As such, we include low-mass dwarf analogs, like HALO6, in this comparison. For $[\mathrm{Fe} / \mathrm{H}]$ values, we only compare stars with metallicity higher than $[\mathrm{Fe} / \mathrm{H}] \sim-3.5$, below which the observation could be incomplete owing to the lack of ability to observe metal-poor stars with current observations.

As shown in Figure 9, we find that the maximum metallicities of the simulated galaxies, $[\mathrm{Fe} / \mathrm{H}]_{\max } \sim-1.8$ (HALO2) and $[\mathrm{Fe} / \mathrm{H}]_{\max } \sim-1.5$ (HALO3), are likely to be consistent with the most metal-rich stars among the observed UFDs, such as CVn II, Leo IV, Coma Berenices, Bootes I, and Hercules. The absence of high-metallicity stars above $[\mathrm{Fe} / \mathrm{H}] \sim-1.5$ in such UFDs, in combination with the enhanced $[\alpha / \mathrm{Fe}]$ ratios, could imply that these UFDs might have experienced similar SFHs to the two galaxies, HALO2 and HALO3, that show the early quenching of star formation by reionization. This interpretation is in line with that of Brown et al. (2014), who report that five UFDs, CVn II, Leo IV, Bootes I, Hercules, and CVn II, show early truncated star formation based on the old ages of their stellar populations, finding that $80 \%$ of their stars formed prior to $z=6$. Here we have demonstrated for the first time that the theoretically expected chemical evolution of UFD analogs formed in a cosmological framework is consistent with this early truncation of star formation.

In addition to the excellent agreement of $[\alpha / \mathrm{Fe}]$ ratios of Bootes I with those of HALO3, the coexistence of CEMP and $\mathrm{C}$-normal stars can be reproduced in HALO3, in which CEMPs are likely to be formed via Population III SNe, while C-normal stars are a consequence of self-enrichment by Population II stars. On the other hand, Segue 1 appears to be similar to
HALO4, providing good agreement between the observed and simulated chemical properties such as $[\mathrm{Fe} / \mathrm{H}]_{\max } \sim$ $-1.42,[\mathrm{C} / \mathrm{Fe}]$, and $[\alpha / \mathrm{Fe}]$. As pointed out in Section 3.4.2, the star with $[\mathrm{C} / \mathrm{Fe}]=1.4$ at $[\mathrm{Fe} / \mathrm{H}]=-1.6$ might originate from a binary system (Frebel et al. 2014). Therefore, we could ignore this outlier since binary systems are not considered in this work. We find that HALO4 exhibits bursty star formation at late epochs at $z \sim 3.9$, during which time stars are formed with relatively high metallicities $([\mathrm{Fe} / \mathrm{H}] \sim-1.6)$. This suggests that star formation in Segue 1 might be not completely truncated by reionization, but followed by late bursts of star formation that gives rise to stars with $[\mathrm{Fe} / \mathrm{H}] \gtrsim-1.6$. We find that none of the simulated UFD analogs exhibit chemical properties similar to those of UMa I.

For example, the $[\alpha / \mathrm{Fe}]$ ratios of UMa II are consistent with the derived $[\alpha / \mathrm{Fe}]$ ratios of HALO6, which is a halo that shows extended star formation beyond reionization. However, HALO6 fails to match $[\mathrm{Fe} / \mathrm{H}]_{\max }=-1$ of UMa II (Gilmore et al. 2013), by producing stars with metallicity higher than $[\mathrm{Fe} / \mathrm{H}] \sim-1$. This discrepancy might indicate that reionization was insufficient to completely suppress star formation in UMa II, allowing it to continuously form stars after reionization, but afterward it is clear that star formation was truncated by other factors, i.e., gas stripping during the infall into the MW halo. The simulations suggest that without this truncation UMa II might have continuously or episodically formed stars down to $z=0$, producing stars with metallicity above $[\mathrm{Fe} / \mathrm{H}] \sim-1$, as predicted in HALO6.

If we assume that the end of star formation of UMa II is instead correlated with the infall time into the MW halo, the simulated analog, HALO6, allows us to estimate the infall time of UMa II. In Figure 11, we present the evolution of chemical abundances of HALO6, $[\alpha / \mathrm{Fe}]$ versus $[\mathrm{Fe} / \mathrm{H}]$, at different redshifts, $z=7$ (top), $z=6$ (middle), and $z=3$ (bottom), with those of UMa II at $z=0$. As shown in the middle panel of Figure 11 , the larger value of $[\mathrm{Fe} / \mathrm{H}]_{\max } \sim-1$ of the most metal-rich stars in UMa II, compared to that of HALO6, indicates an incapability of complete quenching of star formation by reionization. As stars in HALO6 are continuously formed, the metallicity increases, eventually forming stars with metallicity as high as $[\mathrm{Fe} / \mathrm{H}] \sim-1$ at $z \sim 3$. Provided that there are no observed stars with metallicity higher than $[\mathrm{Fe} / \mathrm{H}] \sim-1$ in UMa II, our simulations indicate that its SFH was truncated at $z \sim 3$.

If we assume that the infall onto the MW was responsible for the quenching of star formation in UMa II, we can estimate that UMa II fell in $\sim 11$ Gyr ago. This prediction is consistent with the results of Rocha et al. (2012), where they investigate the infall times for MW dwarfs using their present-day kinematics and the energy-infall relation. They suggest that UMa II tends to support an early infall with $t_{\text {infall }} \sim 8-11 \mathrm{Gyr}$.

We note that it is impossible to infer the accurate infall times of the observed UFDs from this work, since the simulated galaxies are in the field, which is beyond the virial radius of the MW-like host halo. Furthermore, based on the ongoing star formation and the substantial amount of gas reservoir at $z=0$, HALO6 should be considered as a low-mass dwarf rather than UFDs. However, this simulation could provide useful guidance on estimating the time when SFH of UFDs is truncated based on the maximum stellar metallicity observed. 

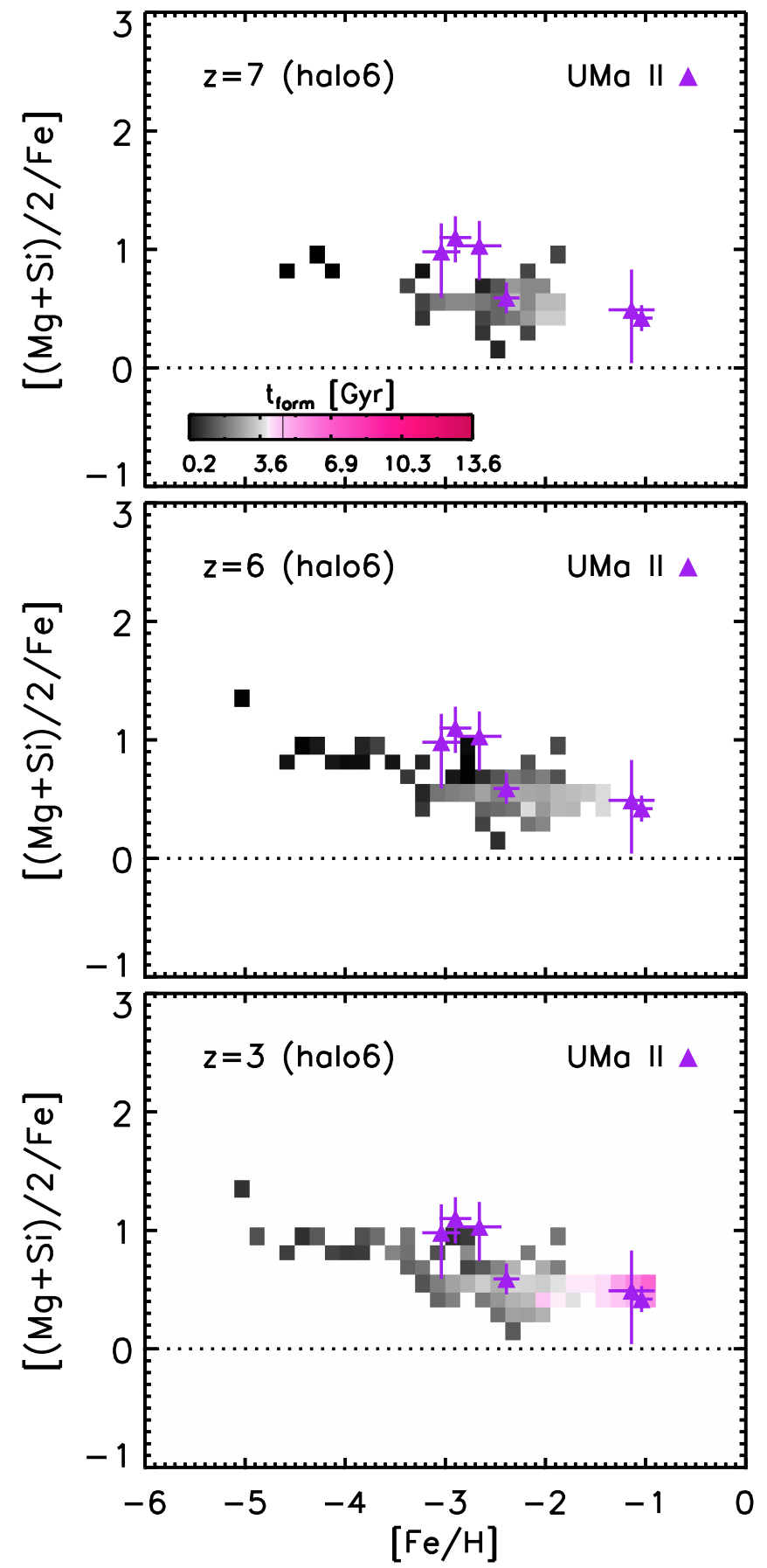

Figure 11. Evolution of stellar abundances, $[\alpha / \mathrm{Fe}]$ vs. $[\mathrm{Fe} / \mathrm{H}]$, in HALO6 at different redshifts, $z=7$ (top), $z=6$ (middle), and $z=3$ (bottom), by comparing with the observed values of UMa II at $z=0$ (Gilmore et al. 2013). As demonstrated in the middle panel, the discrepancy in $[\mathrm{Fe} / \mathrm{H}]_{\max } \sim-1$ between HALO6 and UMa II could indicate that reionization was insufficient to completely quench star formation in UMa II. HALO6 forms stars with such metallicities at $z=3$ (bottom panel), meaning that UMa II formed stars down to $z=3$ and truncated afterward. Furthermore, if we assume that the end of star formation of UMa II is correlated with gas stripping while falling onto the MW halo, then the HALO6 results suggest an estimated infall time for UMa II that is as old as $\sim 11$ Gyr ago.

\subsection{Global Galaxy Properties}

We present global galaxy properties at $z=0$ inferred from the simulations in Figure 12. The relationship between stellar and halo mass of galaxies is shown in the top panel, where the
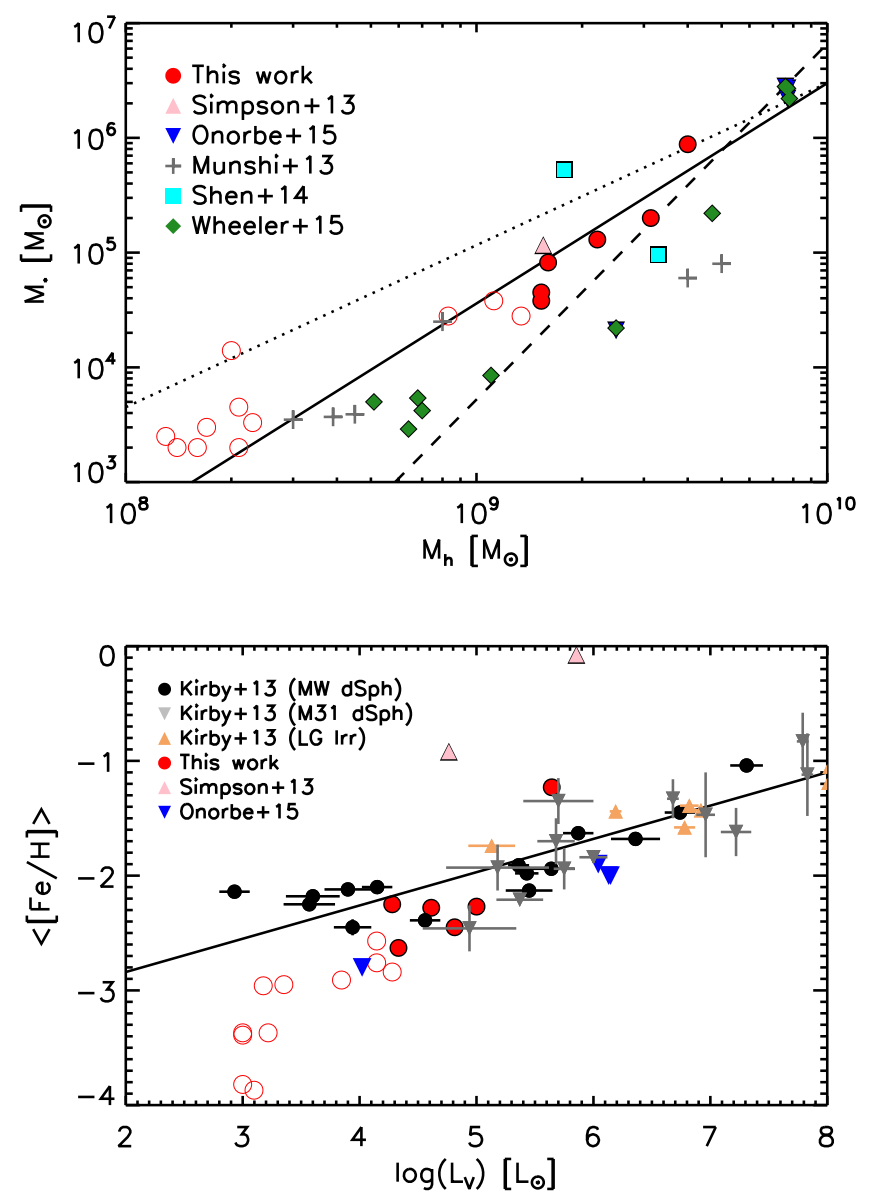

Figure 12. Global physical properties of the simulated dwarf galaxies at $z=0$, stellar mass vs. halo mass (top) and stellar metallicity vs. luminosity (bottom). The quantities derived from our work are shown as red filled circles. We also show results from other hydrodynamic simulations (Munshi et al. 2013; Simpson et al. 2013; Shen et al. 2014; Oñorbe et al. 2015; Wheeler et al. 2015), as well as select observations (Kirby et al. 2013). In the top panel, we compare our stellar mass vs. halo mass results to theoretical fits from abundancematching techniques (Behroozi et al. 2013 [dotted line]; Brook et al. 2014 [dashed line]; Garrison-Kimmel et al. 2016 [solid line]); as can be seen, our results show good agreement with the fit suggested by Garrison-Kimmel et al. (2016). We also mark the properties of low-mass galaxies $\left(M_{\text {halo }}<10^{9} M_{\odot}(z=0)\right.$; red open circles) that are located around the six galaxies (HALO1-HALO6). These galaxies are composed of only a small number of stellar clusters formed via external metal enrichment, giving rise to low average metallicity.

red filled circles denote our simulations. Results from other works are also displayed, including hydrodynamic simulations (e.g., Munshi et al. 2013; Simpson et al. 2013; Shen et al. 2014; Oñorbe et al. 2015; Wheeler et al. 2015) and abundance matching using DM simulations (e.g., Behroozi et al. 2013; Brook et al. 2014; Garrison-Kimmel et al. 2016). Given that our spatial and mass resolutions are comparable to Wheeler et al. (2015) and Oñorbe et al. (2015), the discrepancy in the predicted stellar masses might be attributed to additional feedback effects considered there. Specifically, they also take into account the impact of photoheating and radiation pressure from stars, which could reduce star formation.

Our results show good agreement with a fit, $M_{*} \propto M_{\text {vir }}^{1.8}$, suggested by Garrison-Kimmel et al. (2016), who derived the relation based on LG galaxy counts and the results of DM simulations. The average stellar metallicity versus $V$-band luminosity is presented in the bottom panel of Figure 12 and 
compared again with other works and observations. The $V$ band luminosity is computed by assuming a stellar mass-tolight ratio of $2 M_{\odot} / L_{\odot}$ for an old stellar population (Kruijssen \& Mieske 2009). The average stellar metallicity ranges from $[\mathrm{Fe} / \mathrm{H}]=-1.23$ for a $5 \times 10^{5} L_{\odot}$ galaxy to $[\mathrm{Fe} / \mathrm{H}]=-2.63$ for $2 \times 10^{4} L_{\odot}$ systems, which is in good agreement with observations.

In addition to the six halos (HALO1-HALO6), we also present the properties of small galaxies $\left(M_{\text {halo }}<10^{9} M_{\odot}(z=0)\right.$; red open circles) around the six halos in the refined regions. A noticeable discrepancy from the observed relation is that such small galaxies result in much smaller stellar metallicity, by an order of magnitude, at luminosities below $L_{\mathrm{V}} \sim 10^{3.5} L_{\odot}$. This corresponds to systems in which only a few stars are formed by external metal enrichment, and where subsequent star formation is self-terminated by $\mathrm{SN}$ feedback, evacuating all remaining gas. Afterward, these systems are unable to replenish their gas supply, owing to their shallow potential well. As a result, those low-mass galaxies are composed of only a small number of stellar clusters that form in externally enriched environments, giving rise to low average metallicity. However, the robustness of the derived properties of such small galaxies is subject to mass resolution. Therefore, we plan to firm up this possible external enrichment pathway with even higher-resolution simulations in future work.

\section{Caveats and Limitations}

In this section, we briefly comment on the limitations of this work and our adopted assumptions that could affect the results. One of the robust results of our work is the key role of external metal enrichment in the formation of extremely metal-poor stars. The degree of external metal enrichment, however, heavily depends on how we implement the transport of metals and their mixing with the surrounding gas. In the diffusion method, adopted here, the efficiency of mixing is determined by the diffusion coefficient described in Section 2.4.3.

To test the robustness of our results regarding the efficiency of metal mixing, we additionally perform a comparison simulation. Here we artificially reduce the diffusion coefficient by a factor of 5 in the HALO1 zoom-in simulation and examine whether we still can produce extremely low metallicity stars. We find that the fraction of stars, defined as $f=$ (number of stars with metallicity less than a given $[\mathrm{Fe} / \mathrm{H}]) /($ total number of stars $)$, decreases for $f([\mathrm{Fe} / \mathrm{H}]<-3), \mathrm{f}([\mathrm{Fe} / \mathrm{H}<-4])$, and $f([\mathrm{Fe} / \mathrm{H}]<-5)$ by a factor of $1.5,1.2$, and 1.04, respectively. Given the stochastic nature of star formation, we conclude that the ability of producing low-metallicity stars within our simulations is not very sensitive to changes in the diffusion coefficient. We note that external metal enrichment is argued to be much less important in the recent work by Griffen et al. (2016), where they investigate the fossils of Population III star formation and of the first galaxies in an MW-mass system. The idealized model for metal pollution, implemented in postprocessing within their DM-only simulation, however, makes it difficult to directly compare with their results.

Another uncertainty that could affect the resulting chemical abundances would be the IMF of Population III and Population II stars. As previously mentioned, the Population III IMF, in particular, is still very uncertain. Here we only consider the mass range between 10 and $150 M_{\odot}$ by assuming a top-heavy IMF, but recent high-resolution studies of Population III star formation have suggested that the mass of primordial stars could extend to as low as $m_{*}<1 M_{\odot}$ (e.g., Stacy et al. 2016). If this were the case, our simulations would have overproduced metals, and overpredicted alpha-element abundances as well, via CCSNe and PISNe from massive Population III stars. In addition, the low SFRs in the simulated galaxies indicate that IMF sampling is likely to be incomplete. However, this stochastic IMF sampling is a reasonable assumption for Population III clusters. This is because Population III stars tend to be formed as single or multiple stars, and the following energetic SNe prevent further star formation in small halos $\left(M_{\mathrm{vir}} \lesssim 10^{7} M_{\odot}\right.$ at $\left.z>10\right)$. Therefore, it is expected that the chemical abundances of Population II stars are predominately determined by the yields from one or a few Population III SNe.

We do not take into account photoheating and radiation pressure from stars. For the low-mass galaxies simulated here, these additional stellar feedback effects may play an important role in suppressing star formation. In this context, Wise et al. (2012) have reported that the SFR can be reduced by a factor of five in a dwarf galaxy at $z \sim 8$ with a virial mass of $2 \times 10^{8} M_{\odot}$ by including radiation pressure. Similarly, the key role of radiative feedback in predicting the stellar masses of galaxies, in particular at high redshifts, is emphasized in the study by Hopkins et al. (2014). However, it is currently still prohibitive computationally to trace the evolution of a galaxy down to $z=0$, while directly solving the radiative transfer equation for our highly resolved star-forming regions within their large-scale cosmological context.

It should also be pointed out that we fix the onset of reionization by introducing an external UV background at $z=7$, ramping it up to its full strength by $z=6$. Simpson et al. (2013), on the other hand, have shown that, depending on the onset of reionization, the resultant stellar mass at $z=0$ can vary by an order of magnitude (see also Milosavljević \& Bromm 2014). The precise time when a given galaxy is impacted by the UV background would depend on how far the galaxy is located from massive halos, where the majority of UV photons are emitted, implying that dwarf galaxies close to a massive system might experience an early truncation of star formation. In addition, apart from the uniform UV background, local heating and ionization from the nearby galaxy are neglected. The effect of local radiation sources and the impact of the MW potential will be presented in a subsequent paper. Recently, Oñorbe et al. (2017) have provided a new set of UV backgrounds to complement the extensively used heating and ionization rates (Haardt \& Madau 2012), which turn out to be far stronger at early epochs $(z>6)$, heating the temperature of the IGM to $10^{4} \mathrm{~K}$ already at $z=13$. This means that star formation in the simulated galaxies could have terminated at earlier times than it should, resulting in the overestimated stellar mass at $z=0$. However, we expect that our results may be insensitive to this difference because we introduce the UV background from $z=7$, where two models begin to produce similar reionization history and the IGM temperature.

The final point is that the size of our simulation box is relatively small, dictated by the need to keep computational cost under control. We cannot, therefore, properly represent the large-scale modes in the cosmological density field that would control the number of structures at $z=0$ and would also affect the accretion history of the simulated halos. However, in this work we focus on the evolution of individual systems rather than the statistics of substructures of a realistic MW-size halo. 
In addition, performing simulations for multiple refined regions allows us to investigate the buildup of galaxies under different accretion histories, thus partially addressing the impact of cosmic variance.

\section{Summary and Conclusions}

We have performed a suite of cosmological hydrodynamic zoom-in simulations to investigate the SFH and chemical evolution of local low-mass dwarf galaxies. We have, for the first time, traced the detailed enrichment history of stellar populations in UFD galaxies, reaching back to the formation of Population III stars. Our work, therefore, allows us to directly compare the derived stellar chemical abundances, including the contribution from the first stars, to the detailed observations of individual stellar abundances in UFDs. Given the physical characteristics of the local UFDs, which consist of metal-poor, ancient stellar populations ( $\gtrsim 10 \mathrm{Gyr})$, there have been theoretical attempts to link the nearby UFDs to galaxies at high redshifts $(z \gtrsim 6)$. In particular, the truncated SFH of UFDs, inferred from observations, supports the idea that their star formation could be partially or completely quenched by reionization. The gas in dwarf galaxies was then photoheated, and the filtering (Jeans) mass in the reionized IGM was increased, such that gas collapse into low-mass halos became inefficient.

Our simulations confirm that reionization, in combination with SN feedback, did play a critical role in suppressing star formation in local dwarf galaxies. The effectiveness of reionization in preventing star formation strongly depends on halo mass: star formation in low-mass halos, with $M_{\text {vir }} \lesssim 2 \times 10^{9} M_{\odot}(z=0)$, is almost entirely quenched by reionization, while relatively massive halos, $M_{\text {vir }} \gtrsim 3 \times 10^{9} M_{\odot}(z=0)$, exhibit a continuous or bursty SFH at late epochs (e.g., our HALO6). We find that in most cases more than $90 \%$ of stars form prior to reionization, such that these systems can be classified as "true fossils." On the other hand, the most massive halo, HALO6, with $M_{\text {vir }} \sim 4 \times 10^{9} M_{\odot}(z=0)$, forms only about $30 \%$ of stars before reionization, and star formation is not effectively shut down. Such a galaxy would correspond to a "polluted fossil" in terms of the definition of Ricotti \& Gnedin (2005). The comparison run without $\mathrm{SN}$ feedback confirms that this feedback is essential in truncating star formation in dwarf galaxies.

The ability to resolve small halos $\left(M_{\mathrm{vir}}<10^{8} M_{\odot}\right.$ at $\left.z>6\right)$ enables us to track down stellar mass growth in minihalo scales at high $z$, leading to very interesting results. Our simulations find that star formation in the simulated galaxies, especially less massive halos $\left(M_{\mathrm{vir}}<2 \times 10^{9} M_{\odot}\right.$ at $\left.z=0\right)$, is a consequence of the combination of in situ star formation in a primary halo and significant stellar accretion through multiple mergers of smaller halos, which originate from low-density peaks. This conflicts with the prevailing view that stellar mass growth is dominated by in situ SF within a dominant primary haloinstead, it is difficult to identify a primary halo at early times for UFD analogs. This suggests that when we observe stars in UFDs it is not simple to infer where the stars originate, complicating efforts to reconstruct their SFHs. On the other hand, the most massive halo, HALO6, presents the expected trend that more than $80 \%$ of stars are formed in a primary halo. This case is because the progenitor halos of HALO6 were from high-density peaks and the primary halo grows fast through accretion and mergers. This suggests that there is a distinction between the assembly history of UFDs and that of low-mass dwarfs.

Our simulations demonstrate that the inclusion of Population III $\mathrm{SNe}$ is a necessary ingredient to produce low-metallicity stars with $[\mathrm{Fe} / \mathrm{H}] \lesssim-4$. Particularly, we find that the existence of such low-metallicity stars is predominantly a consequence of external metal enrichment by Population III $\mathrm{SNe}$. Due to the proximity between halos at high redshifts, $z \gtrsim 7$, metals from Population III SNe can be transferred into neighboring halos that have never formed stars but are about to do so. As a result, extremely low metallicity Population II stars are likely to be born in this externally contaminated environment. Based on the distinctive trends of metal yields from Population III and Population II SNe, we scrutinize the detailed origin of the stellar components in the dwarfs. We show that the simulated galaxies are composite systems, assembled from halos that were externally enriched, hosting only Population II stars, and from halos in which the gas was polluted via selfenrichment, hosting both Population III and Population II star formation. Our simulations naturally reproduce the CEMP stars by including Population III SNe that intrinsically yield high ratios of $[\mathrm{C} / \mathrm{Fe}]$. Also, the resultant chemical abundances provide a good match to the observations, in that we find the enhanced $[\alpha / \mathrm{Fe}]$ ratios at all metallicities, a trend also found in the observations of UFDs. This enhanced $[\alpha / \mathrm{Fe}]$ ratio, nearly constant with metallicity, implies that the gas in the dwarfs is more likely affected by Type II rather than Type Ia SNe.

Comparing the derived chemical properties of the simulated galaxies with those of the observed UFDs (CVn II, Leo IV, Coma Berenices, Bootes I, Hercules, Segue I, UMa I, and UMa II), we find that all these UFDs appear to have experienced truncated star formation. Especially, the chemical properties of four UFDs, CVn II, Hercules, Coma Berenices, and Bootes I, tend to be consistent with those of the simulated analogs, HALO2 and HALO3, possibly implying that star formation in these UFDs was entirely quenched by reionization. The presence of relatively high metallicity stars in Leo IV and Segue I might be associated with bursts of star formation at late epochs after reionization, as suggested in HALO4 and HALO5.

The most massive dwarf halo in our sample, HALO6 $\left(\mathrm{M}_{\mathrm{vir}} \sim 4 \times 10^{9} M_{\odot}\right.$ at $\left.z=0\right)$, continues forming stars until $z=0$ and retains a significant neutral $\mathrm{H} \mathrm{I}$ reservoir at $z=0$. The amount of neutral hydrogen and stellar mass at $z=0$ is comparable to that of newly discovered gas-rich, low-mass dwarfs like Leo A, Leo P, Leo T, and DDO 210. This work indicates that the progenitors of such gas-rich dwarfs are slightly more massive than true fossil relics of reionization. This study indicates for the first time the significance of the chemical evolution of low-mass halos to $z=0$ in a full cosmological context to make direct comparison with the full variety of dependent dwarfs observed around the MW. In particular, if we assume that this quenching of star formation in UMa II was correlated with gas stripping while infalling onto the MW halo, the estimated infall time of UMa II could be $t_{\text {infall }} \sim 11 \mathrm{Gyr}$. However, HALO6 already looks very different from UFDs $\left(M_{*}<10^{5} M_{\odot}\right.$ at $\left.z=0\right)$, forming $30 \%$ of stars after reionization, giving rise to a stellar mass of $M_{*}=2.6 \times 10^{5} M_{\odot}(z=6)$, meaning that there likely is a hard upper limit on the UFD halo mass. It also suggests that 
UMa I and UMa II could be the most massive UFDs among the observed UFDs, but still not as massive as HALO6.

A key prediction of our simulations is the presence of extremely low metallicity stars, with $[\mathrm{Fe} / \mathrm{H}] \lesssim-4$, in the local UFDs. These stars should preserve pure Population III signatures, i.e., enhanced carbon and alpha abundances, as a consequence of external metal enrichment and the buildup of dwarfs via mergers. To date, such extremely low metallicity stars have not yet been detected in UFDs, but they should come within reach over the next decade, with the advent of a number of next-generation telescopes. Among them is the Large Synoptic Survey Telescope, with its unprecedented wide-field imaging capability, ideally suited for detecting low surface brightness systems. In addition, the greatly improved spectroscopic sensitivities of the upcoming extremely large groundbased telescopes, the Giant Magellan Telescope, the Thirty Meter Telescope, and the European Extremely Large Telescope, will revolutionize chemical abundance studies in the LG. Also, a recent study of the most metal-poor damped Ly $\alpha$ (DLA) system at $z \sim 3$, with an iron abundance of $[\mathrm{Fe} / \mathrm{H}] \lesssim-2.81$, suggests that the chemical signatures from a core-collapse SN of a $20.5 M_{\odot}$ Population III star are imprinted on the observed DLA, indicating that such systems could be the predecessors of UFDs (Cooke et al. 2017). All these avenues promise to offer fundamental insights into the nature of the first generation of stars and into the metal enrichment history of the early universe.

We are grateful to Volker Springel, Joop Schaye, and Claudio Dalla Vecchia for letting us use their versions of GADGET and their data visualization and analysis tools. We thank Jun-Hwan Choi for discussions of the simulation setup. The authors would like to thank the referee for constructive comments that significantly improved the quality of the manuscript. The simulations were performed with the Texas Advanced Computing Center (TACC) at the University of Texas at Austin for providing HPC resources under XSEDE allocation TG-AST160038 (PI M. Jeon). The authors acknowledge the El Gato cluster at the University of Arizona, which is funded by the National Science Foundation through grant no. 1228509. V.B. was supported by NSF grant AST-1413501.

\section{References}

Abel, T., Anninos, P., Zhang, Y., \& Norman, M. L. 1997, NewA, 2, 181 Abel, T., Bryan, G. L., \& Norman, M. L. 2002, Sci, 295, 93

Aoki, W., Beers, T. C., Christlieb, N., et al. 2007, ApJ, 655, 492

Barris, B. J., \& Tonry, J. L. 2006, ApJ, 637, 427

Beers, T. C., \& Christlieb, N. 2005, ARA\&A, 43, 531

Behroozi, P. S., Wechsler, R. H., \& Conroy, C. 2013, ApJ, 770, 57

Bland-Hawthorn, J., Sutherland, R., \& Webster, D. 2015, ApJ, 807, 154

Bovill, M. S., \& Ricotti, M. 2009, ApJ, 693, 1859

Bovill, M. S., \& Ricotti, M. 2011, ApJ, 741, 18

Boylan-Kolchin, M., Weisz, D. R., Johnson, B. D., et al. 2015, MNRAS, 453, 1503

Bromm, V. 2013, RPPh, 76, 112901

Bromm, V., Coppi, P. S., \& Larson, R. B. 2002, ApJ, 564, 23

Bromm, V., Kudritzki, R. P., \& Loeb, A. 2001, ApJ, 552, 464

Bromm, V., \& Yoshida, N. 2011, ARA\&A, 49, 373

Brook, C. B., Di Cintio, A., Knebe, A., et al. 2014, ApJL, 784, L14

Brown, T. M., Tumlinson, J., Geha, M., et al. 2012, ApJL, 753, L21

Brown, T. M., Tumlinson, J., Geha, M., et al. 2014, ApJ, 796, 91

Bullock, J. S., Kravtsov, A. V., \& Weinberg, D. H. 2000, ApJ, 539, 517

Chatzopoulos, E., \& Wheeler, J. C. 2012, ApJ, 748, 42

Cooke, R. J., \& Madau, P. 2014, ApJ, 791, 116
Cooke, R. J., Pettini, M., \& Steidel, C. C. 2017, MNRAS, 467, 802

Corlies, L., Johnston, K. V., Tumlinson, J., \& Bryan, G. 2013, ApJ, 773, 105

Dalla Vecchia, C., \& Schaye, J. 2012, MNRAS, 426, 140

Durier, F., \& Dalla Vecchia, C. 2012, MNRAS, 419, 465

Fan, X., Strauss, M. A., Becker, R. H., et al. 2006, AJ, 132, 117

Ferland, G. J., Korista, K. T., Verner, D. A., et al. 1998, PASP, 110, 761

Fitts, A., Boylan-Kolchin, M., Elbert, O. D., et al. 2017, MNRAS, 471, 3547

Förster, F., Wolf, C., Podsiadlowski, P., \& Han, Z. 2006, MNRAS, 368, 1893

Frebel, A., \& Bromm, V. 2012, ApJ, 759, 115

Frebel, A., Norris, J. E., Gilmore, G., \& Wyse, R. F. G. 2016, ApJ, 826, 110

Frebel, A., Simon, J. D., Geha, M., \& Willman, B. 2010, ApJ, 708, 560

Frebel, A., Simon, J. D., \& Kirby, E. N. 2014, ApJ, 786, 74

Gardner, J. P., Mather, J. C., Clampin, M., et al. 2006, SSRv, 123, 485

Garrison-Kimmel, S., Bullock, J. S., Boylan-Kolchin, M., \& Bardwell, E. 2017, MNRAS, 464, 3108

Gilmore, G., Norris, J. E., Monaco, L., et al. 2013, ApJ, 763, 61

Glover, S. C. O., \& Jappsen, A.-K. 2007, ApJ, 666, 1

Gnedin, N. Y., \& Kravtsov, A. V. 2006, ApJ, 645, 1054

Governato, F., Weisz, D., Pontzen, A., et al. 2015, MNRAS, 448, 792

Grebel, E. K., \& Gallagher, J. S., III, 2004, ApJL, 610, L89

Greif, T. H., Johnson, J. L., Klessen, R. S., \& Bromm, V. 2009, MNRAS, 399, 639

Griffen, B. F., Dooley, G. A., Ji, A. P., et al. 2016, arXiv:1611.00759

Gunn, J. E., \& Peterson, B. A. 1965, ApJ, 142, 1633

Haardt, F., \& Madau, P. 2011, arXiv:1103.5226

Haardt, F., \& Madau, P. 2012, ApJ, 746, 125

Hahn, O., \& Abel, T. 2011, MNRAS, 415, 2101

Haiman, Z., Thoul, A. A., \& Loeb, A. 1996, ApJ, 464, 523

Heger, A., \& Woosley, S. E. 2002, ApJ, 567, 532

Heger, A., \& Woosley, S. E. 2010, ApJ, 724, 341

Hirano, S., Hosokawa, T., Yoshida, N., et al. 2014, ApJ, 781, 60

Hirano, S., Hosokawa, T., Yoshida, N., Omukai, K., \& Yorke, H. W. 2015, MNRAS, 448, 568

Hopkins, P. F., Kereš, D., Oñorbe, J., et al. 2014, MNRAS, 445, 581

Hosokawa, T., Hirano, S., Kuiper, R., et al. 2016, ApJ, 824, 119

Hosokawa, T., Omukai, K., Yoshida, N., \& Yorke, H. W. 2011, Sci, 334, 1250

Ishigaki, M. N., Aoki, W., Arimoto, N., \& Okamoto, S. 2014, A\&A, 562, A146

Iwamoto, N., Umeda, H., Tominaga, N., Nomoto, K., \& Maeda, K. 2005, Sci, 309,451

Jeon, M., Pawlik, A. H., Bromm, V., \& Milosavljević, M. 2014, MNRAS, 444, 3288

Ji, A. P., Frebel, A., \& Bromm, V. 2015, MNRAS, 454, 659

Kirby, E. N., Cohen, J. G., Guhathakurta, P., et al. 2013, ApJ, 779, 102

Kitayama, T., \& Yoshida, N. 2005, ApJ, 630, 675

Klessen, R. S., \& Lin, D. N. 2003, PhRvE, 67, 046311

Komatsu, E., Smith, K. M., Dunkley, J., et al. 2011, ApJS, 192, 18

Kruijssen, J. M. D., \& Mieske, S. 2009, A\&A, 500, 785

Kudritzki, R.-P., \& Puls, J. 2000, ARA\&A, 38, 613

Lapi, A., Mancuso, C., Celotti, A., \& Danese, L. 2017, ApJ, 835, 37

Mannucci, F., Della Valle, M., \& Panagia, N. 2006, MNRAS, 370, 773

Marigo, P. 2001, A\&A, 370, 194

McConnachie, A. W. 2012, AJ, 144, 4

McKee, C. F., \& Tan, J. C. 2008, ApJ, 681, 771

McQuinn, K. B. W., Skillman, E. D., Dolphin, A., et al. 2015, ApJ, 812, 158

Meynet, G., Ekström, S., \& Maeder, A. 2006, A\&A, 447, 623

Milosavljević, M., \& Bromm, V. 2014, MNRAS, 440, 50

Munshi, F., Governato, F., Brooks, A. M., et al. 2013, ApJ, 766, 56

Muratov, A. L., Gnedin, O. Y., Gnedin, N. Y., \& Zemp, M. 2013, ApJ, 773, 19

Norris, J. E., Yong, D., Bessell, M. S., et al. 2013, ApJ, 762, 28

Omukai, K. 2000, ApJ, 534, 809

Oñorbe, J., Boylan-Kolchin, M., Bullock, J. S., et al. 2015, MNRAS, 454, 2092

Oñorbe, J., Hennawi, J. F., \& Lukić, Z. 2017, ApJ, 837, 106

Planck Collaboration, 2016, A\&A, 594, A13

Portinari, L., Chiosi, C., \& Bressan, A. 1998, A\&A, 334, 505

Revaz, Y., Arnaudon, A., Nichols, M., Bonvin, V., \& Jablonka, P. 2016, A\&A, 588, A21

Revaz, Y., Jablonka, P., Sawala, T., et al. 2009, A\&A, 501, 189

Ricotti, M., \& Gnedin, N. Y. 2005, ApJ, 629, 259

Ricotti, M., Parry, O. H., \& Gnedin, N. Y. 2016, ApJ, 831, 204

Ritter, J. S., Safranek-Shrader, C., Gnat, O., Milosavljević, M., \& Bromm, V. 2012, ApJ, 761, 56

Rocha, M., Peter, A. H. G., \& Bullock, J. 2012, MNRAS, 425, 231

Safranek-Shrader, C., Montgomery, M. H., Milosavljević, M., \& Bromm, V. 2016, MNRAS, 455, 3288 
Saitoh, T. R., \& Makino, J. 2009, ApJL, 697, L99

Salvadori, S., \& Ferrara, A. 2009, MNRAS, 395, L6

Salvadori, S., Skúladóttir, Á, \& Tolstoy, E. 2015, MNRAS, 454, 1320

Sawala, T., Scannapieco, C., Maio, U., \& White, S. 2010, MNRAS, 402, 1599

Schaerer, D. 2002, A\&A, 382, 28

Schmidt, M. 1959, ApJ, 129, 243

Schneider, R., \& Omukai, K. 2010, MNRAS, 402, 429

Shen, S., Madau, P., Conroy, C., Governato, F., \& Mayer, L. 2014, ApJ, 792, 99

Simon, J. D., Frebel, A., McWilliam, A., Kirby, E. N., \& Thompson, I. B 2010, ApJ, 716, 446

Simpson, C. M., Bryan, G. L., Johnston, K. V., et al. 2013, MNRAS, 432, 1989

Smith, B. D., Wise, J. H., O'Shea, B. W., Norman, M. L., \& Khochfar, S. 2015, MNRAS, 452, 2822

Springel, V. 2005, MNRAS, 364, 1105

Springel, V., White, S. D. M., Tormen, G., \& Kauffmann, G. 2001, MNRAS, 328,726

Stacy, A., Bromm, V., \& Lee, A. T. 2016, MNRAS, 462, 1307

Stacy, A., Greif, T. H., \& Bromm, V. 2012, MNRAS, 422, 290
Susa, H., Hasegawa, K., \& Tominaga, N. 2014, ApJ, 792, 32

Tegmark, M., Silk, J., Rees, M. J., et al. 1997, ApJ, 474, 1

Thielemann, F.-K., Argast, D., Brachwitz, F., et al. 2003, in From Twilight to Highlight: The Physics of Supernovae, ed. W. Hillebrandt \& B. Leibundgut (Berlin: Springer), 331

Tolstoy, E., Hill, V., \& Tosi, M. 2009, ARA\&A, 47, 371

Umeda, H., \& Nomoto, K. 2003, Natur, 422, 871

Vargas, L. C., Geha, M., Kirby, E. N., \& Simon, J. D. 2013, ApJ, 767, 134

Webster, D., Bland-Hawthorn, J., \& Sutherland, R. 2015, ApJL, 799, L21

Webster, D., Sutherland, R., \& Bland-Hawthorn, J. 2014, ApJ, 796, 11

Weisz, D. R., \& Boylan-Kolchin, M. 2017, MNRAS, 469, L83

Weisz, D. R., Dolphin, A. E., Skillman, E. D., et al. 2014, ApJ, 789, 148

Wetzel, A. R., Deason, A. J., \& Garrison-Kimmel, S. 2015, ApJ, 807, 49

Wheeler, C., Oñorbe, J., Bullock, J. S., et al. 2015, MNRAS, 453, 1305

Wiersma, R. P. C., Schaye, J., \& Smith, B. D. 2009a, MNRAS, 393, 99

Wiersma, R. P. C., Schaye, J., Theuns, T., Dalla Vecchia, C., \& Tornatore, L. 2009b, MNRAS, 399, 574

Wise, J. H., Turk, M. J., Norman, M. L., \& Abel, T. 2012, ApJ, 745, 50

Yong, D., Norris, J. E., Bessell, M. S., et al. 2013, ApJ, 762, 27 